\title{
Deep Sequencing the microRNA profile in rhabdomyosarcoma reveals down-regulation of miR-378 family members
}

\author{
Francesca Megiorni ${ }^{1 *+}$, Samantha Cialfi ${ }^{1,2+}$, Heather P McDowell ${ }^{1,3,4}$, Armando Felsani ${ }^{5}$, Simona Camero ${ }^{1}$, \\ Alessandro Guffanti ${ }^{5}$, Barry Pizer ${ }^{3}$, Anna Clerico ${ }^{1}$, Alessandra De Grazia ${ }^{1}$, Antonio Pizzuti ${ }^{6}$, Anna Moles ${ }^{5}$ \\ and Carlo Dominici ${ }^{1,4}$
}

\begin{abstract}
Background: Rhabdomyosarcoma (RMS) is a highly malignant tumour accounting for nearly half of soft tissue sarcomas in children. MicroRNAs (miRNAs) represent a class of short, non-coding, regulatory RNAs which play a critical role in different cellular processes. Altered miRNA levels have been reported in human cancers, including RMS.

Methods: Using deep sequencing technology, a total of 685 miRNAs were investigated in a group of alveolar RMSs (ARMSs), embryonal RMSs (ERMSs) as well as in normal skeletal muscle (NSM). Q-PCR, MTT, cytofluorimetry, migration assay, western blot and immunofluorescence experiments were carried out to determine the role of miR-378a-3p in cancer cell growth, apoptosis, migration and differentiation. Bioinformatics pipelines were used for miRNA target prediction and clustering analysis.

Results: Ninety-seven miRNAs were significantly deregulated in ARMS and ERMS when compared to NSM. MiR-378 family members were dramatically decreased in RMS tumour tissue and cell lines. Interestingly, members of the miR-378 family presented as a possible target the insulin-like growth factor receptor 1 (IGF1R), a key signalling molecule in RMS. MiR-378a-3p over-expression in an RMS-derived cell line suppressed IGF1R expression and affected phosphorylated-Akt protein levels. Ectopic expression of miR-378a-3p caused significant changes in apoptosis, cell migration, cytoskeleton organization as well as a modulation of the muscular markers MyoD1, MyoR, desmin and MyHC. In addition, DNA demethylation by 5-aza-2'-deoxycytidine (5-aza-dC) was able to up-regulate miR-378a-3p levels with a concomitant induction of apoptosis, decrease in cell viability and cell cycle arrest in G2-phase. Cells treated with 5-aza-dC clearly changed their morphology and expressed moderate levels of MyHC.
\end{abstract}

Conclusions: MiR-378a-3p may function as a tumour suppressor in RMS and the restoration of its expression would be of therapeutic benefit in RMS. Furthermore, the role of epigenetic modifications in RMS deserves further investigations.

Keywords: Rhabdomyosarcoma, MicroRNAs, Deep sequencing, miR-378a-3p, 5-aza-2'-deoxycytidine

\section{Background}

Rhabdomyosarcoma (RMS) is the most common soft tissue sarcoma in childhood [1], representing approximately $50 \%$ of all sarcomas in children aged $0-14$ years and $4-5 \%$ of malignant solid tumours in the paediatric population. The two major histological subtypes, alveolar

\footnotetext{
* Correspondence: francesca.megiorni@uniroma1.it

${ }^{\dagger}$ Equal contributors

'Department of Paediatrics and Infantile Neuropsychiatry, Sapienza University of Rome, Viale Regina Elena 324, 00161 Rome, Italy

Full list of author information is available at the end of the article
}

rhabdomyosarcoma (ARMS) and embryonal rhabdomyosarcoma (ERMS), have distinct clinical features and outcomes. ERMSs are more frequent ( $80 \%$ of cases) and generally affect younger children ( $0-4$ years); they occur more commonly in the neck, head and genito-urinary tract [2]. As the name implies, tumour cells resemble embryonal skeletal muscle cells. ARMSs ( $\sim 20 \%$ of cases) usually present throughout childhood, typically originating in the limbs and trunk, often with regional or metastatic lymph node involvement already at diagnosis, and carry a significantly worse outcome $[2,3]$. ARMS is so

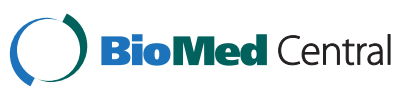


called because tumour cells form small spaces or pseudoalveoli.

The role of genetic factors in the development of RMS has been confirmed by several recent epidemiological observations and advances in molecular genetics. Although the majority of RMS cases are sporadic, i.e. not associated with hereditary syndromes, a small proportion are linked to congenital anomalies, e.g. BeckwithWiedemann syndrome, or are associated with particular familial syndromes, such as neurofibromatosis type I and Li-Fraumeni syndrome [4-6]. ARMS and ERMS are both characterised by particular genetic alterations that are likely to play a decisive role in cancer pathogenesis. Eighty percent of ARMS tumours have either $t(2 ; 13)$ $(\mathrm{q} 35, \mathrm{q} 14)$ or $\mathrm{t}(1 ; 13)(\mathrm{p} 36 ; \mathrm{q} 14)$ chromosomal translocations, which generate PAX3-FOXO1 and PAX7-FOXO1 fusion proteins, respectively [7]. However, constitutive expression of PAX3/7-FOXO1 chimeric genes is not sufficient to induce RMS development in transgenic animals $[8,9]$. Loss of heterozygosity of the short arm of chromosome 11 (11p15.5), with over-expression of the insulin-like growth factor II, is often associated with ERMS [10]. However, although several tumour causative genes have been identified, a detailed understanding of the molecular mechanisms underlying RMS development has not yet been achieved.

Recent studies have suggested that microRNAs (miRNAs) may play an essential role in RMS [11,12]. MiRNAs are a class of conserved, short, non-coding molecules which regulate gene expression through binding to nonperfect complementary sequences at the 3 '-untranslated regions (UTRs) of target messenger RNAs (mRNAs), resulting in translational repression [13]. Several premiRNAs stem-loops are processed to produce two mature and functional miRNAs, designated with the suffix "-3p" or "-5p," depending on the originating hairpin arm [14]. It has been predicted that about one-third of all mammalian genes are targeted by miRNAs [15,16]. Deregulation of miRNA expression is associated with various cancers and many studies indicate that miRNAs may act either as oncogenes or tumour suppressors [17-19], controlling key processes in tumorigenesis, such as tumour initiation, progression and metastatic spread. An increasing number of miRNAs, such as miR-1, miR-133a, miR-200c, miR-206, miR-214 and miR-9*, have been identified to have a role in RMS [11,20-24], as recently summarized by Novak et al. [12].

Epigenetic DNA changes, such as DNA promoter hypermethylation and histone modifications, have critical roles in chromatin remodelling and general regulation of gene expression in mammalian development and human diseases [25]. In particular, DNA methylation of CpG islands in promoter regions has been correlated with silencing of tumour suppressor genes and other tumour-related genes, and it has been recognised as a crucial component of the mechanism underlying cancer development [26]. DNA methylation-associated silencing of miRNAs in different human tumours, including RMS, has also been reported $[27,28]$.

In this study, deep sequencing technology was utilised to quantify the absolute abundance of miRNAs in ARMS and ERMS tumours as well as in normal skeletal muscle (NSM), and to identify an RMS-specific miRNA expression pattern. The majority of miRNAs were found to be downregulated, as predicted. Interestingly, miR-378a/b/c/d/e/f/ $\mathrm{h} / \mathrm{i}$ molecules, belonging to a large family of evolutionary conserved miRNAs, were strongly under-represented in ARMS and ERMS tumours in comparison to NSM. Transient transfection of miR-378a-3p in an ARMSderived cell line (RH30) induced apoptosis and decreased viability/proliferation by repressing the IGF1R/AKT pathway. Importantly, elevated levels of miR-378a-3p impaired RH30 cell migration and promoted myogenic differentiation. Demonstration that epigenetic modifications may be involved in RMS tumourigenesis was achieved by restoring miR-378a-3p levels. In addition, 5-aza-2'-deoxycytidine (5-aza-dC) treatment induced apoptosis, cell cycle arrest in G2 phase and decreased cell viability compared to untreated RH30 cells. Interestingly, RH30 5-aza-dC-treated cells changed their morphology and expressed muscle differentiation markers, partially overlapping the effect of miR-378a-3p transfection.

Taken together, these data provide the first evidence for an anti-tumour activity of miR-378a-3p in RMS, suggesting that this miRNA could be a potential therapeutic target in RMS. Furthermore, the importance of epigenetic regulation in RMS was confirmed, which may have important clinical implications in this malignancy.

\section{Methods \\ Patient clinical and tumour histopathological characteristics}

Fifteen RMS tumour samples, 7 ARMSs and 8 ERMSs, were obtained at diagnosis before any treatment from children admitted to the Department of Paediatrics and Infantile Neuropsychiatry at "Sapienza" University, and to the Department of Oncology at Alder Hey Children's NHS Trust, Liverpool. Histopathological diagnosis was confirmed using immunohistochemistry. All 7 ARMS were investigated for PAX3/7-FOXO1 translocations using standard FISH analysis: 5 tumours were PAX3FOXO1-positive, 1 was PAX7-FOXO1-positive and 1 was fusion-negative. Patients were grouped according to the Intergroup Rhabdomyosarcoma Study (IRS) postsurgical grouping system [29]. Details of the patients are described in Table 1. ARMS1-2-3-4 and ERMS1-2-3-4 tumour samples were used for deep sequencing study. Institutional written informed consent was obtained from 
Table 1 Clinico-pathological features of the analysed tumour cases

\begin{tabular}{|c|c|c|c|c|}
\hline Case & Histology & Fusion status & Primary site & Clinical stage \\
\hline ARMS1* & alveolar & PAX3 & trunk & III \\
\hline ARMS2* & alveolar & PAX3 & extremity & III \\
\hline ARMS3* & alveolar & negative & trunk & $\|$ \\
\hline ARMS4* & alveolar & PAX7 & uterus-vagina & III \\
\hline ARMS7 & alveolar & PAX3 & extremity & IV \\
\hline ARMS36 & alveolar & PAX3 & trunk & III \\
\hline ARMS37 & alveolar & PAX3 & uterus-vagina & $\|$ \\
\hline ERMS1* & embryonal & n.a. & retroperitoneum & III \\
\hline ERMS2* & embryonal & n.a. & bladder-prostate & III \\
\hline ERMS3* & embryonal & n.a. & bladder-prostate & III \\
\hline ERMS4* & embryonal & n.a. & trunk & III \\
\hline ERMS12 & embryonal & n.a. & bladder-prostate & III \\
\hline ERMS21 & embryonal & n.a. & retroperitoneum & III \\
\hline ERMS23 & embryonal & n.a. & uterus-vagina & $\|$ \\
\hline ERMS27 & embryonal & n.a. & extremity & I \\
\hline
\end{tabular}

Variables were categorized as follows: histological subtype, embryonal versus alveolar; gene fusion status; primary site and clinical stage. Fusion status PAX3: PAX3-FOXO1-positive; PAX7: PAX7-FOXO1-positive; n.a. - not applicable. The symbol * indicates samples used for deep sequencing analysis.

the patient's parents or legal guardians. The study underwent ethical review and approval according to the local institutional guidelines (Policlinico Umberto I's Ethics Committee and Alder Hey Children's NHS Foundation Trust Ethics Committee). Control RNA was extracted from normal skeletal muscle (NSM) obtained from eight children undergoing surgery for benign conditions.

\section{RNA isolation}

Samples were immediately frozen in liquid nitrogen after surgery and stored at $-80^{\circ} \mathrm{C}$. Total RNA was extracted using TRIzol (Invitrogen) according to the manufacturer's instructions. Samples were enriched for small RNAs up to 200 bp by size selection using Pure Link miRNA Isolation Kit (LifeTech). RNA purity, integrity and size distribution were assessed using an Agilent 2100 Bioanalyzer (Agilent Technologies).

\section{Small RNA library generation and sequencing}

Enriched RNA samples were processed using the Small RNA Expression Kit according to the manufacturer's protocol (Small RNA expression kit, rev. C, Applied Biosystems). Briefly, RNA was first hybridized and ligated with the adapter mix "A", subsequently reverse transcribed and treated with RNAse $\mathrm{H}$. The obtained cDNA libraries were PCR amplified, purified and size-selected by PAGE, resulting in libraries containing inserted small RNA sequences of 20-40 bp length. Size, integrity and purity of the libraries were verified by the Agilent 2100
Bioanalyzer (Agilent Technologies). cDNA libraries were barcoded using the Solid RNA barcoding kit and amplified onto beads using emulsion PCR. Templated beads were deposited on slides and analysed using the Applied Biosystems SOLiD 4 Sequencer.

\section{Statistical and bioinformatics analyses}

The quality filtered reads were mapped against all annotated human mature miRNA sequences (miRBase v19.0) [30] using the Lifetech Lifescope 2.5.1 Small RNA pipeline, filtering for rRNA, primers, and small non-miRNA non-coding transcribed sequences such as tRNAs and snoRNAs. Sequence counts were extracted and reformatted with perl scripts from the pipeline output. Differential expression analysis was performed with the edgeR Bioconductor statistical library [31] version 3.0.8 on $R$ version 2.15.3. TMM-normalized sequence counts in the libraries were transformed in Counts Per Millions (CPM) according to the formula: $\mathrm{CPM}=$ (normalized counts/total miRNA matches) " $1,000,000$. After having estimated the tagwise dispersion, genewise exact test [32] as implemented in edge $R$ was used to measure the significance of differential expression, using the gene "Pseudo-counts". Sequences were deemed significantly differentially expressed if (1) the p-value given by this method was $<0.05$, (2) the total count was greater than 50 CPM in at least one group of samples, and (3) there was at least a two-fold change in normalised sequence counts between the two groups.

The validated target prediction of a panel of regulated microRNAs was performed by interrogating the 'Validated Target' option of the miRWalk web software [33]. Target genes of the miR-378 family were predicted using TargetScan Human 6.2 (http://www. targetscan.org/), miRanda (http://www.microrna.org/ microrna/home.do) and DIANA-microT version 3.0 (http://diana.cslab.ece.ntua.gr/microT/) algorithms. Annotation and enrichment of functional pathways associated with the miR-378 target genes were evaluated using the Reactome database and associated analytical tools (http:// www.reactome.org/). The same miR-378 target gene list was used as the starting dataset for the generation of a Functional Interaction network analysis and related Gene Ontology enrichment analysis as described [34,35]. Preliminary isoMIR analysis was performed by selecting from the YM500 miR-Seq database (http://ngs.ym.edu.tw/ ym500v2/index.php) the hsa-miR378a-3p isoforms which had been found with at least 50.000 sequence reads in 40 different experiments, allowing 3 nt $5^{\prime}$ extensions, $3^{\prime}$ extensions and one mismatch with respect to the reference miRBase mature sequence. The resulting 14 isomirs were identified and counted in all the NSM, ARMS and ERMS sequences using the bowtie 0.12 .8 database search software and ad-hoc created perl scripts. 
Nucleotide sequence pattern analysis of the miR-378a$3 p$ promoter region was performed using the program CpG plot of the EMBOSS sequence analysis suite (http://emboss.sourceforge.net/), with standard parameters, and other softwares such as $\mathrm{CpG}$ island searcher (http://cpgislands.usc.edu/) on the genome region chr5: 149107388-149112388 corresponding to the putative has-miR378a-3p putative promoter.

Each experiment was repeated three times independently. All results were expressed as means \pm standard deviation (SD), and a p-value $<0.05$ was used for significance. One-way ANOVA analysis for independent samples was used to determine statistical significance in different assays.

\section{Cell cultures}

Human ARMS RH30 and ERMS RD cell lines were maintained in high-glucose Dulbecco's modified Eagle's medium(DMEM-HG) supplemented with $10 \%$ foetal bovine serum (FBS), 1\% v/v L-glutamine, $100 \mu \mathrm{g} / \mathrm{ml}$ streptomycin and $100 \mathrm{U} / \mathrm{ml}$ penicillin, and grown at $37^{\circ} \mathrm{C}$ in a humidified atmosphere of $5 \% \mathrm{CO}_{2}$.

\section{5-aza-2'-deoxycytidine treatment}

RH30 and RD cells were seeded at $4 \times 10^{5}$ cells/well in 6-well plates. After 24 h, 5-aza-dC (Sigma-Aldrich, St. Louis, MO) was added to a final concentration of $20 \mu \mathrm{M}$. Following different times of treatment, cells were collected for cell cycle analysis, apoptosis, MTT, migration, Q-PCR, western blot or immunofluorescence. Mock treatments were carried out treating cells in the same medium with DMSO (Ctr).

\section{Transient transfection}

RH30 and RD cells were seeded at $8 \times 10^{5}$ cells/well in 6-well plates; miRNA mimics (miR-378a-3p, Dharmacon Research) or negative control (miR-Ctr, Dharmacon Research) were transfected using Lipofectamine 2000 reagent (Life Technologies) at $50 \mathrm{nM}$ final concentration, following the manufacturer's protocol. Following different times of treatment, cells were collected for cell cycle analysis, apoptosis, MTT, migration, Q-PCR, western blot or immunofluorescence.

\section{Quantitative Real Time PCR (Q-PCR)}

Reverse transcription (RT) for human miR-378a-3p, miR-378a-5p, miR-483-3p and miR-503-5p was carried out with TaqMan MicroRNA Assay kit (Life Technologies) using $20 \mathrm{ng}$ of total RNA sample and the specific stem-loop primer according to manufacturer's protocols. Quantitative Real Time PCR (Q-PCR) analysis was performed on a StepOne Real Time System (Life Technologies) machine using miRNA-specific TaqMan MGB primers/probe (Life Technologies). PCR reactions were run at $95^{\circ} \mathrm{C}$ for $10 \mathrm{~min}$, followed by 40 cycles at $95^{\circ} \mathrm{C}$ for $15 \mathrm{~s}$ and $60^{\circ} \mathrm{C}$ for $30 \mathrm{~s}$. Data were normalized to U6 small nuclear RNA (RNU6) levels. Each sample was run in triplicate. The amount of each miRNA was calculated by the comparative $\mathrm{Ct}$ method and expressed as fold change $\left(2^{-\Delta \Delta C T}\right)$ compared to NSM using the DataAssist v3.01 software (Life Technologies).

\section{MTT assay}

RH30 cells were treated with 5 -aza-dC or transfected with miRNA mimics, and cell viability was determined using MTT [3-(4,5-dimethylthiazol-2-yl)-2,5-diphenyltetrazolium] assay. Cells $\left(5 \times 10^{3}\right)$ were plated onto 96-well plates in sextuplicates and, after $24 \mathrm{~h}$, treated with 5-aza-dC or transfected with synthetic miR-378-3p; untreated and blank cell-free controls were included. At designated times after treatment or transfection (0-2448-72 h), $10 \mu \mathrm{l}$ of MTT (5 mg/mL, Sigma-Aldrich) were added to each well and plates were incubated at $37^{\circ} \mathrm{C}$ for $4 \mathrm{~h}$. Media were removed and $150 \mu \mathrm{l}$ dimethyl sulfoxide (DMSO) were added into each well to dissolve the dark blue formazan crystals. Absorbance was measured at wavelength of $550 \mathrm{~nm}$, with reference at $630 \mathrm{~nm}$, using a microtitre plate reader (Select Science). The results were plotted as means \pm SD of two separate experiments having six determinations per experiment for each experimental condition.

\section{Cell cycle analysis}

RH30 and RD cells treated with 5-aza-dC were collected and washed twice with phosphate buffered saline (PBS). After fixation in $70 \%$ ice-cold ethanol overnight at $+4^{\circ} \mathrm{C}$, cell pellets were washed twice with ice-cold PBS and treated with RNase A for $15 \mathrm{~min}$ at $37^{\circ} \mathrm{C}$. Propidium iodide (PI) was added to each sample and DNA content was determined by collecting 10,000 events using a BD FACS Calibur Flow Cytometer (BD Biosciences). Data were analysed using CellQuest Pro software (BD Biosciences). Experiments were performed three times.

\section{Apoptosis analysis}

Cell apoptosis was analysed by flow cytometry with PE Annexin V Apoptosis Detection Kit I (BD Pharmingen). Briefly, RH30 and RD cells were seeded overnight in 6well plate and treated with 5 -aza-dC or transfected with miRNA mimics for $72 \mathrm{~h}$. Cells were washed twice in cold PBS and resuspended in 1x Annexin V Binding Buffer at a concentration of $1 \times 10^{6}$ cells $/ \mathrm{ml}$. Cells were stained with Annexin $\mathrm{V}$ and 7-Amino-Actinomycin D (7-AAD) for $15 \mathrm{~min}$ at room temperature (RT) in darkness according to the manufacturer's instructions. Annexin V and 7-AAD fluorescence intensities of control or treated samples were analysed using a BD FACSCalibur Flow Cytometer (BD Biosciences). Data were 
analysed using Cell Quest Pro software (BD Biosciences). Experiments were performed three times.

\section{Migration assay}

RH30 cells were cultured in complete medium with 5aza-dC or miRNA mimics for $72 \mathrm{~h}$ before plating $5 \times 10^{4}$ cells per well into BD FalconTM Cell Culture Inserts with $8 \mu \mathrm{m}$ pore polycarbonate filters (Falcon). Chambers with cells contained medium without serum, whilst the lower well had DMEM supplemented with 10\% FBS, used as chemoattractant. After $24 \mathrm{~h}$, migrated cells at the base of the inserts were fixed in $100 \%$ methanol and stained with $2 \%$ crystal violet dye. Cells were photographed under a light microscope at $20 \times$ or $40 \times$ magnifications; 8 randomly selected fields were examined and counted manually. The average number of migrated cells was calculated. Experiments were performed in triplicate and repeated twice.

\section{Western blot analysis}

RMS cells were seeded overnight in 6-well plates. Cells were treated with 5-aza-dC or transfected with miRNA mimics for 72-96 $\mathrm{h}$ and lysed with Hepes buffer (20 mM Hepes, $250 \mathrm{mM} \mathrm{NaCl}, 0.1 \%$ Triton X-100, $2 \mu \mathrm{mM}$ EDTA, $10 \mu \mathrm{g} / \mathrm{mL}$ leupeptin, $10 \mu \mathrm{g} / \mathrm{mL}$ aprotinin, $0.5 \mathrm{mM}$ phenylmethylsulfonyl fluoride, $4 \mathrm{mM}$ sodium orthovanadate, $1 \mathrm{mM}$ DTT). Total protein extracts $(30 \mu \mathrm{g})$ were separated on $8-12 \%$ sodium dodecyl sulfate (SDS)polyacrylamide gel (PAGE) and transferred onto polyvinylidene fluoride (PVDF) membranes (Millipore Corporation, Bedford, MA). Filters were blocked with $5 \%$ non-fat dry milk in PBS-Tween for $30 \mathrm{~min}$ at RT and incubated with the primary antibody. The following antibodies were incubated over-night at $+4^{\circ} \mathrm{C}$ : anti-IGFR1 (Cell Signaling), anti-phospho-Akt (Cell Signaling), anti-MyoR(Santa Cruz Biotechnology), anti-MyOD1 (Millipore), anti-Myf5 (Millipore), anti-desmin (Millipore) and anti-MyHC (Millipore). Appropriate horseradish peroxidase (HRP)-conjugated secondary antibodies (Santa Cruz Biotechnology) were used for $1 \mathrm{~h}$ at RT. Protein-antibody complexes were detected with ECL Super Signal (Pierce). Tubulin (Sigma-Aldrich) was used as a normalization control for equal loading. Experiments were performed at least three times.

\section{Immunofluorescence}

RMS cells were seeded overnight in 24-well plates. Cells were treated with 5 -aza-dC or transfected with miRNA mimics for $72 \mathrm{~h}$ and then seeded into 8-chamber culture slides (Falcon). After two additional days, cells were rinsed with PBS and fixed with $4 \%$ paraformaldehyde at RT for $30 \mathrm{~min}$. After treatment with $0.1 \mathrm{M}$ glycine and permeabilisation with $0.1 \%$ Triton $\mathrm{X}-100$, cells were subjected to immunofluorescence staining with the antiMyHC (Millipore) antibody for $1 \mathrm{~h} 30 \mathrm{~min}$ at RT. Cells were washed with cold PBS three times and incubated with Texas Red-anti-mouse secondary antibody (1:100, Jackson Laboratories) at RT for $30 \mathrm{~min}$. The actin cytoskeleton was visualized with TRITC-phalloidin (1:50, Sigma) at RT for $1 \mathrm{~h} 30 \mathrm{~min}$. Nuclei were counterstained with $1 \mu \mathrm{g} / \mathrm{ml}$ Hoechst (Sigma). Labelled sections were examined and analysed by using a Zeiss ApoTom epifluorescent microscope (Carl Zeiss) and Axio-Vision software. Experiments were replicated twice.

\section{DNA Methylation analysis}

Genomic DNA from RH30 cells treated or not with 5-aza$\mathrm{dC}$ for $72 \mathrm{~h}$ was obtained by phenol:chloroform:isoamyl alcohol method. About $1 \mu \mathrm{g}$ of DNA was modified using the Epitect DNA Bisulfite Kit (Qiagen), according to the manufacturer's protocol. For sequencing, the bisulfitetreated DNA was amplified by PCR with two different primer sets for the human miR-378a promoter: BS1 forward 5'-GGGGAAAAGttAGGtTGGA-3' and BS1 reverse 5' -aCTaACATTTTTaaTaaCTaCTTaTCCCAaC-3'; BS2 forward 5'-GGGTAAtTGGGGGTTttAG-3' and BS2 reverse 5'-CAaCAACAaCACTCTaaaACT-3'. PCR products, purified using a commercially available kit (Qiagen), were sequenced on an $\mathrm{ABI}$ sequencer with dye terminators (Applied Biosystems). Analysis of primary bisulfite sequencing was carried out with BISMA software (http://services. ibc.uni-stuttgart.de/BDPC/BISMA) by uploading the unconverted reference sequence and the sequencing results. For methylation-specific PCR (MSP), the bisulfite-modified genomic DNA was amplified using primers based on methylated $(\mathrm{M})$ or unmethylated (UM) cytosines in CpG islands in the miR-378a promoter region: $M$ forward $5^{\prime}$-AGtTAGCGGtttTGCGGtAGtC-3' and M reverse 5'-aCCCGaaaaAaaaAaCCAaCGAaCG-3'; UM forward 5'-tttGtttttGtAGtTAGtGGtttTGtGGtAGttG-3' and UM reverse 5'-CaAaCCaaCCCaaaaaaAaaaAaCCAa $\mathrm{CaAaCa}-3^{\prime}$. PCR products were run on $2 \%$ agarose gel. All primers were designed using the MSPprimer algorithm. Experiments were performed three times.

\section{Results}

Small RNA library generation, sequencing, identification and quantification of annotated miRNAs

As accumulating evidence indicates that miRNAs play important roles in cancer development, including RMS, this study profiled the miRNA transcriptome by the direct sequencing of mature miRNA molecules in a panel of primary RMS tumours. RNA was prepared from four ARMSs and four ERMSs, and from a pool of NSM obtained from eight donors. As RMS has a mainly infiltrative growth pattern, the quantity of available tumour samples is very often a limiting factor, especially for controls and NGS experiments. Hence, we used a set of pre-pooled NSM samples from already available normal 
donors thus averaging out the variance at the expense of some loss in biological variability. However, relevant differential representation of single miRNA molecules stood out clearly in a comparison of individual samples versus a pooled control, minimizing the inter-individual 'transcriptional noise'. Clinical characteristics of the tumour cases are reported in Table 1. Small RNA libraries were prepared and deep sequenced by using a SOLiD4 Sequencer platform. The corresponding nine cDNA libraries yielded a total of 250 million sequenced reads, and more than $85 \%$ of these reads (an average of 22 million per library) mapped against the human reference genome (GRCh37/hg19, repeat masked). The reads corresponding to annotated miRNAs were identified by selecting all reads mapping against the human precursor and mature sequences comprised in miRBase v19.0. After passing alignment quality filtering, from 2 to 7 million reads were identified as annotated miRNAs per library (on average 15\% of hg19 mapped reads), representing 685 different mature miRNA molecules. The abundance value of each target-miRNA was normalized using TMM normalization, scaled to "counts per million" (CPM) in respect to each library size and the associated "pseudo-counts" were used for the differential expression analysis. Figure 1 and Table 2 show the distribution of the different miRNA species in abundance classes comparing ARMS and ERMS samples against NSM, allowing a survey of the whole miRNA population. Apparently, tumour miRNAs on average were more represented in the intermediate abundance class $\left(10^{2}\right.$ $\left.10^{4} \mathrm{CPM}\right)$, whilst they were under-represented in the lowest $\left(1-10^{2} \mathrm{CPM}\right)$ and in the highest abundance $\left(>10^{4}\right.$ CPM) classes. These data support the hypothesis that in these tumours a rearrangement of miRNA expression levels occurred, resulting in a reduction of the levels of some highly expressed miRNAs and a simultaneous slight increase of the expression of many low-abundance miRNA species.

The distribution of miR-387a-3p isomirs between NSM and RMSs was assessed. One YM500 database isoform with an extension at $3^{\prime}$ and a mismatch in the extension (ACTGGACTTGGAGTCAGAAGGCG[C]T) was represented in both NSM and ARMS/ERMS samples, but with a noticeable difference in frequency which will warrant further investigation.

\section{Differentially expressed miRNAs}

Of a total of 685 expressed miRNAs, 97 (14.2\%) displayed significant differential levels collectively in ARMS and ERMS tumours in comparison with NSM. Notably, out of these $97,79(81.4 \%)$ miRNAs were expressed at lower levels in RMSs using the TMM normalization (Table 3A), whilst 18 (18.6\%) were expressed at higher levels (Table 3B). Of note, using a different edgeR normalization method (upper quartile) only produced 4 additional differentially expressed miRNAs, while maintaining the total number of detected small RNAs. Among the identified differentially expressed miRNAs, some have been previously associated with tumour development in RMS [36]. Interestingly, miRNAs belonging to the miR-378 family, recently suggested to be essential in normal skeletal muscle development [37], were markedly down-regulated in both ARMS and ERMS tumours (see Additional file 1: Table S1). MiR-133a, miR-378a-3p, miR378a-5p, miR-483-3p and miR-503-5p were selected as candidates to validate miRNA expression levels in Q-PCR using the eight deep-sequencing-analysed RMSs, seven additional tumour samples (3 ARMSs and 4 ERMSs) along with four different RMS cell lines (RH4 and RH30 ARMS cell lines; and RD and RD18 ERMS cell lines). In

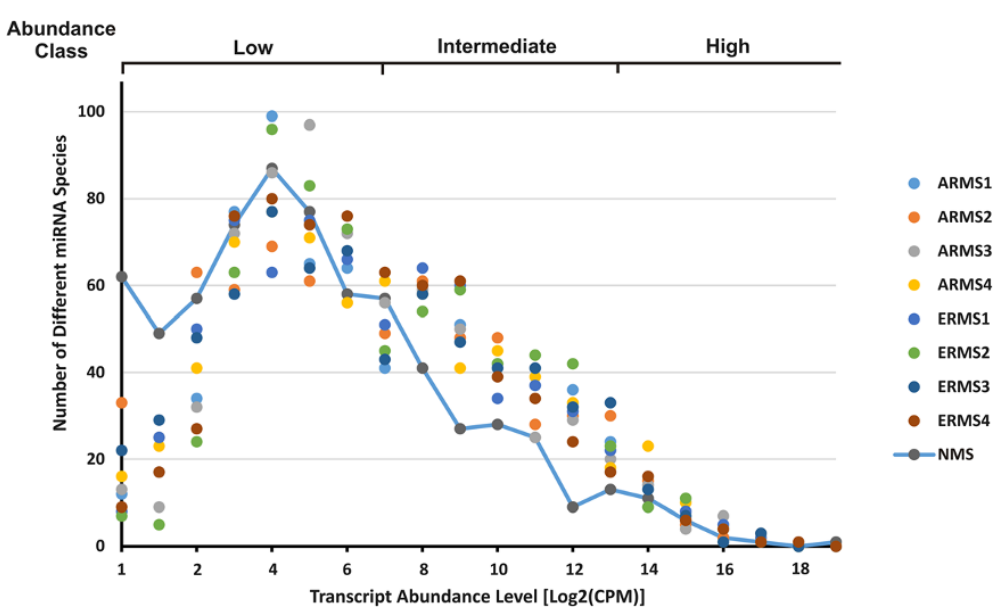

Figure 1 miRNAs in alveolar and embryonal RMS (ARMS and ERMS) samples, together with normal skeletal muscle (NSM). Graphic of the number of different miRNA species in function of transcript expression levels, expressed in Log $_{2}$ CPM, in ARMS, ERMS and NSM samples. Distribution of transcripts in abundance classes (Low < 1-102 CPM; Intermediate $10^{2}-10^{4}$ CPM; High > $10^{4} \mathrm{CPM}$ ) is schematized. 
Table 2 Distribution in abundance classes of different miRNA transcripts in tumours and normal muscle

\begin{tabular}{|c|c|c|c|c|c|c|c|}
\hline \multirow[b]{2}{*}{ CLASSES } & \multirow[b]{2}{*}{ CPM range } & \multicolumn{3}{|c|}{ NMS } & \multicolumn{3}{|c|}{ RHABDOMYOSARCOMAS } \\
\hline & & miRNA species & Cumulative CPM & $\%$ CPM & miRNA species & Cumulative CPM & $\%$ CPM \\
\hline LOW ABUNDANCE & $1-10^{2}$ & 498 & 9,663 & 1.0 & 369 & 9,288 & 0.9 \\
\hline INTERMEDIATE ABUNDANCE & $10^{2}-10^{4}$ & 163 & 214,726 & 21.5 & 296 & 451,671 & 45.2 \\
\hline HIGH ABUNDANCE & $>10^{4}$ & 16 & 775,611 & 77.5 & 20 & 539,041 & 53.9 \\
\hline TOTAL & & 677 & $1,000,000$ & 100.0 & 685 & $1,000,000$ & 100.0 \\
\hline
\end{tabular}

agreement with the deep-sequencing findings, Q-PCR results confirmed the down-regulation of miR-133a, miR378a-3p and miR-378a-5p, as well as the over-expression of miR-483-3p and miR-503-5p in the RMS tumour tissues (see Additional file 2: Figure S1A) and cells (see Additional file 2: Figure S1B). Comparison of deep sequencing ARMS and ERMS data showed a similar range of gene expression profile with the exception of a limited number of miRNAs that displayed significantly different levels (Table 3C).

\section{miR-378a-3p negatively regulates IGF1R levels}

As reported in Table 4, miR-378 family members are transcribed from different loci but they exhibit seed sequence homology for mRNA target recognition. We focused on miR-378a-3p, a key regulatory molecule of miR-378 family, investigating the biological relevance of this miRNA in RMS tumours by performing target gene prediction and functional pathway analysis. TargetScan, DIANA-microT, miRanda and miRWalk algorithms were interrogated, which allowed the identification of a list of putative/validated mRNA targets for miR-378a-3p (see Additional file 3: Table S2). DAVID and Cytoscape Reactome FI, used to functionally cluster the miRNA targeted transcripts and their involvement in various signal pathways, showed a significant enrichment in biological mechanisms and pathways linked with neoplastic diseases and, more specifically, with apoptosis, cell cycle signalling and DNA remodelling/interaction (see Additional file 3: Table S2). Interestingly, the in silico analysis suggested that miR-378a-3p directly participates in the post-transcriptional regulation of IGF1R signalling (Figure 2A), which is involved in the development, growth, proliferation, cell survival and metastasis of RMS [38]. To validate the control of the IGF1R expression, transient transfection ofmiR-378a-3p in RH30 cells, an in vitro model of human ARMS, was carried out. An RNA duplex from C. elegans was used as a negative miRNA-Control (miR-Ctr). Using by stem-loop real-time PCR, specific expression increase of miR-378a-3p after transfection with the respective mimic was demonstrated (see Additional file 4: Figure S2). A down-regulation of the endogenous IGF1R protein levels was observed in miR-378a-3p-transfected RH30 cells (Figure 2B) consistent with the conserved binding sites for this miRNA in the $3^{\prime}$-untranslated region of IGF1R transcript (Figure 2C), identified by computational tools for miRNA target prediction. IGF1R reduction was also confirmed in RD cells transiently transfected with miR-378a-3p (data not shown). These data are also in agreement with those reported by other research groups [39-41] in which the direct regulation of IGF1R mRNA by miR-378a-3p was supported by in vitro luciferase assays. Thus, expression of miR-378a-3p suppresses IGFR1 pathway activity.

miR-378-3p induces apoptosis and impairs cell migration In order to examine the potential anti-oncogenic role of miR-378a-3p in RMS, a series of in vitro gain-offunction experiments were designed. A significant increase of apoptotic cells was observed in alveolar RH30 at $72 \mathrm{~h}$ following transfection of miR-378a-3p mimics $(30.8 \% \pm 1.0)$ in comparison with miR-Ctr $(16.3 \% \pm 2.4)$ (Figure 3A). Similarly, the number of apoptotic cells significantly increased in miR-378a-3p transfected embryonal RD cells versus miR-Ctr positive cells $(24.1 \% \pm 2.9$ vs. $11.2 \% \pm 1.9, \mathrm{p}<0.01)$. Consistent with the FACS results, a decrease of AKT phosphorylation levels and a concomitant increase of cleaved-caspase-3, an important regulator of apoptosis, were detected in miR-378a-3p positive cells compared to scrambled control-treated RH30 cells (Figure 3B). Only a moderate alteration (about 20\%) in cellular viability/proliferation rate was evident in miR-378a-3p-transfected RH30 cultures (Figure 3C). Ectopic expression of miR-378a-3p significantly suppressed by about $60 \%$ the ability of RH30 cells to migrate through Boyden chamber membranes towards serum-containing medium when compared with a mimic negative control (Figure 3D). These results indicate that the restoration of miR-378a-3p levels enhances programmed cell death and inhibits cell viability and migration potentials of RMS cells.

\section{miR-378a-3p correlates with myogenic differentiation}

Expression analysis was carried out using a panel of muscle markers in RH30 cells transfected with miR-378a$3 p$ in order to assess whether miRNA up-regulation was able to promote skeletal muscle differentiation. The modifications found in specific myogenic marker levels were consistent with the induction of myogenic differentiation both in immunoblotting and immunofluorescence 
Table 3 miRNA expression in RMS tumours

\begin{tabular}{|c|c|c|c|c|c|c|c|c|}
\hline \multicolumn{6}{|l|}{$A$} & \multicolumn{3}{|l|}{ B } \\
\hline miRNA_ID & $\log _{2} F C$ & $\log _{2} C P M$ & miRNA_ID & $\log _{2} \mathrm{FC}$ & $\log _{2} C P M$ & miRNA_ID & $\log _{2} \mathrm{FC}$ & $\log _{2} C P M$ \\
\hline hsa-miR-885-5p & -6.93 & 7.34 & hsa-miR-4256 & -2.95 & 9.13 & hsa-miR-4327 & 9.95 & 3.61 \\
\hline hsa-miR-144-5p & -5.62 & 8.27 & hsa-miR-218-5p & -2.95 & 11.07 & hsa-miR-508-3p & 9.63 & 3.40 \\
\hline hsa-miR-451a & -5.52 & 15.36 & hsa-miR-126-3p & -2.93 & 13.30 & hsa-miR-383 & 7.99 & 6.17 \\
\hline hsa-miR-1 & -5.47 & 16.81 & hsa-miR-5787 & -2.93 & 3.93 & hsa-miR-503-5p & 5.68 & 9.48 \\
\hline hsa-miR-378a-5p & -5.34 & 11.64 & hsa-miR-22-5p & -2.88 & 9.77 & hsa-miR-154-3p & 5.19 & 10.09 \\
\hline hsa-miR-223-3p & -5.12 & 12.89 & hsa-miR-30e-3p & -2.81 & 9.06 & hsa-miR-493-5p & 4.89 & 11.33 \\
\hline hsa-miR-124-3p & -5.06 & 5.79 & hsa-miR-139-5p & -2.79 & 9.02 & hsa-miR-450a-5p & 4.88 & 9.11 \\
\hline hsa-miR-378a-3p & -4.92 & 13.79 & hsa-miR-30b-3p & -2.77 & 3.39 & hsa-miR-500b & 4.68 & 9.25 \\
\hline hsa-miR-378f & -4.80 & 7.02 & hsa-miR-203a & -2.72 & 5.26 & hsa-miR-335-3p & 4.60 & 9.51 \\
\hline hsa-miR-378 h & -4.53 & 4.42 & hsa-miR-185-5p & -2.71 & 8.80 & hsa-miR-376c-5p & 4.59 & 4.14 \\
\hline hsa-miR-378c & -4.47 & 12.37 & hsa-miR-652-3p & -2.68 & 7.09 & hsa-miR-483-3p & 4.55 & 13.10 \\
\hline hsa-miR-599 & -4.39 & 4.93 & hsa-miR-4484 & -2.64 & 3.45 & hsa-miR-376a-2-5p & 4.55 & 5.55 \\
\hline hsa-miR-144-3p & -4.36 & 7.41 & hsa-miR-208b & -2.63 & 5.74 & hsa-miR-503-3p & 4.55 & 4.22 \\
\hline hsa-miR-378i & -4.29 & 6.47 & hsa-miR-24-1-5p & -2.60 & 5.69 & hsa-miR-3182 & 4.43 & 7.92 \\
\hline hsa-miR-378d & -4.29 & 8.81 & hsa-miR-30a-3p & -2.60 & 8.81 & hsa-miR-542-3p & 4.36 & 10.77 \\
\hline hsa-miR-486-5p & -4.14 & 11.78 & hsa-miR-29a-3p & -2.59 & 13.25 & hsa-miR-149-5p & 4.33 & 11.39 \\
\hline hsa-miR-133a & -4.05 & 14.25 & hsa-miR-16-2-3p & -2.55 & 8.81 & hsa-miR-501-5p & 3.81 & 11.03 \\
\hline hsa-miR-95 & -3.96 & 9.35 & hsa-miR-628-3p & -2.54 & 2.47 & hsa-miR-483-5p & 3.48 & 9.68 \\
\hline hsa-miR-378b & -3.86 & 5.55 & hsa-miR-29b-2-5p & -2.54 & 5.75 & & & \\
\hline hsa-miR-29c-5p & -3.82 & 7.35 & hsa-miR-133b & -2.53 & 11.85 & $\mathrm{C}$ & & \\
\hline hsa-miR-378e & -3.82 & 9.31 & hsa-miR-4300 & -2.51 & 4.76 & miRNA_ID & $\log _{2} F C$ & $\log _{2} C P M$ \\
\hline hsa-miR-142-5p & -3.68 & 8.68 & hsa-miR-491-5p & -2.32 & 2.56 & hsa-miR-335-5p & -3.25 & 12.19 \\
\hline hsa-miR-4461 & -3.66 & 2.84 & hsa-miR-873-3p & -2.31 & 3.06 & hsa-miR-489 & -3.30 & 7.64 \\
\hline hsa-miR-378 g & -3.64 & 4.58 & hsa-miR-3687 & -2.31 & 2.75 & hsa-miR-375 & -4.11 & 8.33 \\
\hline hsa-miR-944 & -3.56 & 2.73 & hsa-miR-5095 & -2.27 & 4.86 & hsa-miR-135a-5p & -5.38 & 10.49 \\
\hline hsa-miR-486-3p & -3.54 & 6.75 & hsa-miR-29b-1-5p & -2.26 & 3.58 & hsa-miR-9-3p & -6.07 & 6.89 \\
\hline hsa-miR-29c-3p & -3.54 & 12.27 & hsa-miR-664a-5p & -2.25 & 3.52 & hsa-miR-9-5p & -7.53 & 8.95 \\
\hline hsa-miR-4306 & -3.53 & 3.61 & hsa-miR-664a-3p & -2.24 & 7.74 & hsa-miR-499a-3p & 6.21 & 7.83 \\
\hline hsa-miR-4516 & -3.51 & 3.57 & hsa-miR-4315 & -2.22 & 4.39 & hsa-miR-499a-5p & 6.07 & 9.97 \\
\hline hsa-miR-193b-5p & -3.51 & 6.08 & hsa-miR-3714 & -2.21 & 2.64 & hsa-miR-432-5p & 3.58 & 9.47 \\
\hline hsa-miR-139-3p & -3.49 & 2.94 & hsa-miR-4787-3p & -2.18 & 2.50 & hsa-miR-296-5p & 3.37 & 8.53 \\
\hline hsa-miR-616-5p & -3.41 & 4.29 & hsa-miR-7-5p & -2.14 & 6.83 & hsa-miR-95 & 3.30 & 7.89 \\
\hline hsa-miR-22-3p & -3.39 & 9.05 & hsa-miR-7-1-3p & -2.13 & 7.81 & hsa-miR-31-5p & 3.22 & 8.09 \\
\hline hsa-miR-150-5p & -3.33 & 10.54 & hsa-miR-7-2-3p & -2.06 & 3.97 & & & \\
\hline hsa-miR-548v & -3.27 & 3.04 & hsa-miR-23c & -1.98 & 9.82 & & & \\
\hline hsa-miR-3117-3p & -3.05 & 1.97 & hsa-miR-584-5p & -1.96 & 2.87 & & & \\
\hline hsa-miR-628-5p & -3.03 & 5.41 & hsa-miR-137 & -1.95 & 5.54 & & & \\
\hline hsa-miR-1281 & -3.02 & 3.73 & hsa-miR-3607-5p & -1.64 & 10.85 & & & \\
\hline hsa-miR-548z & -3.01 & 3.45 & hsa-miR-935 & -1.59 & 5.49 & & & \\
\hline hsa-miR-126-5p & -3.00 & 12.54 & & & & & & \\
\hline
\end{tabular}

(A) miRNA species expressed at lower levels in RMSs vs. NSM (ARMS and ERMS are collectively considered); (B) miRNA species expressed at higher levels in RMSs vs. NSM (ARMS and ERMS are collectively considered); (C) differentially expressed miRNAs in ERMS vs. ARMS tumours. 
Table 4 Human miR-378 family members

\begin{tabular}{|c|c|c|c|c|c|}
\hline miRNA_ID & Accession & Sequence & Locus (strand) & Start & End \\
\hline hsa-miR-378a-3p & MIMAT0000732 & ACUGGACUUGGAGUCAGAAGG & Chr5 (+) & 149112388 & 149112453 \\
\hline hsa-miR-378b & MIMAT0014999 & ACUGGACUUGGAGGCAGAA & Chr3 (+) & 10371913 & 10371969 \\
\hline hsa-miR-378c & MIMAT0016847 & ACUGGACUUGGAGUCAGAAGAGUGG & Chr10 (-) & 132760851 & 132760931 \\
\hline hsa-mir-378d-1 & MIMAT0018926 & ACUGGACUUGGAGUCAGAAA & Chr4 (-) & 5925002 & 5925055 \\
\hline hsa-mir-378d-2 & MIMAT0018926 & ACUGGACUUGGAGUCAGAAA & Chr8 (-) & 94928250 & 94928347 \\
\hline hsa-miR-378e & MIMAT0018927 & ACUGGACUUGGAGUCAGGA & Chr5 (+) & 169455492 & 169455570 \\
\hline hsa-miR-378f & MIMAT0018932 & ACUGGACUUGGAGCCAGAAG & Chr1 (+) & 24255560 & 24255637 \\
\hline hsa-miR-378 g & MIMAT0018937 & ACUGGGCUUGGAGUCAGAAG & Chr1 (-) & 95211416 & 95211456 \\
\hline hsa-miR-378 h & MIMAT0018984 & ACUGGACUUGGUGUCAGAUGG & Chr5 (+) & 154209018 & 154209100 \\
\hline hsa-miR-378i & MIMAT0019074 & ACUGGACUAGGAGUCAGAAGG & Chr22 (-) & 42319226 & 42319301 \\
\hline hsa-miR-378j & MIMAT0024612 & ACUGGAUUUGGAGCCAGAA & Chr17 (-) & 35974976 & 35975084 \\
\hline hsa-miR-378a-5p & MIMAT0000731 & CUCCUGACUCCAGGUCCUGUGU & Chr5 (+) & 149112388 & 149112453 \\
\hline
\end{tabular}

MiRBase (Release 21, June 2014) identifiers, accession numbers, sequence and genome location of human miR-378 family members. The presence of a shared common seed is evident from the aligned sequences.

experiments, performed $96-120 \mathrm{~h}$ after miR-378a-3p induction (Figure 4). In particular, RH30 cells transfected with miR-378a-3p mimics showed a slight upregulation of MyoD1 and $\mathrm{MyHC}$ proteins, which are respectively detected in committed proliferating myoblasts and in post-mitotic muscle cells, with a concomitant down-regulation of MyoR, a repressor of myogenesis, and Myf5, an helix-loop-helix transcription factor correlated with myoblast proliferation stage (Figure 4A). Myoid differentiation was also confirmed by the up-regulation of the intermediate filament desmin, the contractile protein actin expressed in skeletal muscle cells (Figure 4A). Furthermore, an increased number of miR-378a-3p transfected cells exhibited changes in morphology/cytoskeleton organization and moderate levels of MyHC staining (Figure 4B). In particular, more organized actin filaments were observed in miR-378a-3p positive cells, whilst actin appeared more diffusely distributed through-out the cytoplasm in mocked RH30 control cells, as documented by TRITCphalloidin staining (Figure 4B). MyHC immunofluorescence staining had stronger intensity also in $\mathrm{RD}$ cells transfected with miR-378a-3p than miR-Ctr (data not shown). These experiments suggest that miR-378a-3p molecules are involved in the reactivation of terminal myogenic differentiation in RMS by coordinating specific regulatory factors and repressors.

\section{5-aza-2'-deoxycytidine (5-aza-dC) treatment up-regulates miR-378a-3p levels and induces apoptosis, G2-cell arrest and decreased migration in RMS cells}

Since epigenetic modifications have been involved in miRNA deregulation, treatment of RH30 and RD cells with 5 -aza-dC, a demethylating drug, was carried out to see if it could have a direct effect on miR-378a-3p levels given the presence of $\mathrm{CpG}$ islands in its promoter region
(Figure 5A). Incremental dosing of 5-aza-dC (2-10$20 \mu \mathrm{M})$ resulted in up-regulation of the abundance of mature miR-378a-3p molecules compared with the control after $24 \mathrm{~h}$ (Figure 5B). A similar miR-378a-3p increment (about 3.5 fold-change) was observed in $\mathrm{RD}$ treated-cells (data not shown). In this investigation, we analysed the miR-378a-3p methylation pattern in RH30 cells, either untreated or treated with 5 -aza-dC for $72 \mathrm{~h}$. DNA was subjected to bisulfite sequencing (BS) and methylation-specific PCR (MS-PCR). Surprisingly, we had no evidence of methylated $\mathrm{CpG}$ islands either in Ctr (upper line) than in 5-aza-dC-treated cells (lower line) by sequencing BS1 and BS2 miR-378a-3p promoter regions (Figure $5 \mathrm{C}$ ). Unmethylated pattern was also suggested by PCR positivity using primer sets specific for the miR-378a-3p unmethylated CpG (UM) but not with methylated CpG (M) specific primers (Figure 5D). The addition of $20 \mu \mathrm{M} 5$-aza-dC was able to efficiently trigger apoptosis in alveolar RH30 cells $(36.7 \% \pm 3.6 v$ s. $12.4 \% \pm 2.2$ ) (Figure 6A), whilst the amount of apoptotic cells was less in 5-aza-dC embryonal RD cells versus mocked control cells $(26.1 \% \pm 3.8$ vs. $12.1 \% \pm 2.0, \mathrm{p}<$ $0.05)$. The migration capacity of the 5 -aza-dC-treated RH30 cells was significantly diminished compared with a paired negative control (Figure 6B). Furthermore, a significant growth/cell cycle arrest of RH30 cells was evident after drug exposure. The addition of the demethylating drug resulted in the appearance of a small number of living cells with a different morphology compared to untreated control cells. A time-dependent inhibition of cell proliferation resulted in a peak of $80 \%$ at $72 \mathrm{~h}$ post-treatment in RH30 cells (Figure 6C). Similarly, 5 -aza-dC reduced migration and proliferation rates of $\mathrm{RD}$ cells of about $50 \%$ (data not shown). To determine whether growth inhibition was associated with specific 


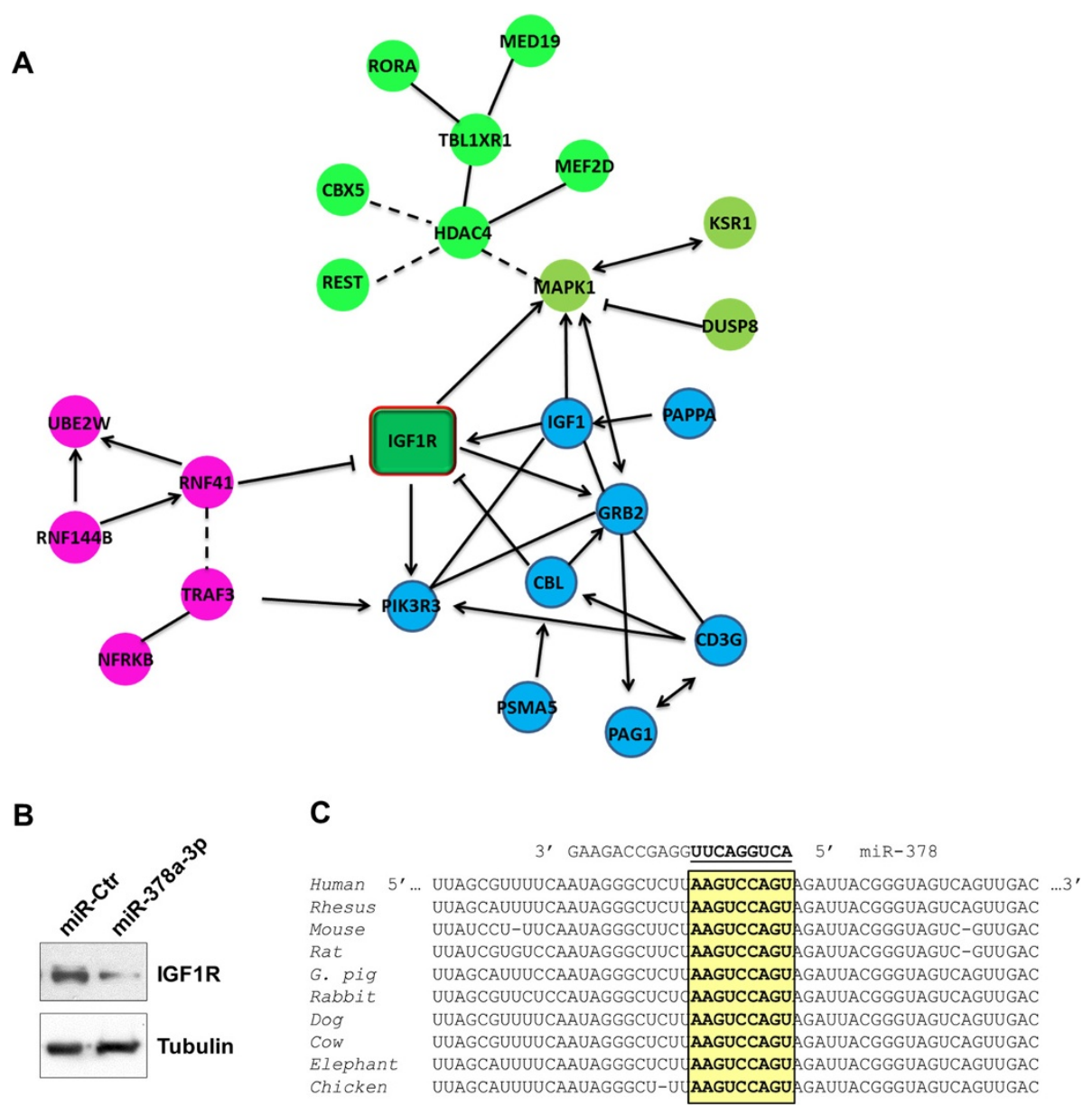

Figure 2 miR-378a-3p negatively regulates IGF1R. (A) Schematic network representation of miR-378a-3p target genes functional annotations and interactions obtained from Cytoscape Reactome FI interrogation. IGF1R represents the principal mRNA target of miR-378a-3p. Putative mRNA targets of miR-378a-3p are predicted to be involved in several signal pathways, specifically apoptosis, cell cycle and chromatin remodelling. Arrow (edge) orientation indicates positive, negative or undetermined functional interaction between different genes (network nodes). Target gene colours indicate different functional modules of the interaction network. (B) Immunoblot analysis. Validation of miR-378a-3p direct participation in the post-transcriptional regulation of IGF1R in RH30 cells (miR-control vs. miR-378a-3p positive cells). Tubulin was used as loading control. (C) Sequence alignment of miR-378a-3p seed sequence (highlighted) with IGF1R 3'-UTR binding sites (bold letters in the yellow box; position 5589-5596 of human IGF1R: NM_000875) in different species. Data from TargetScan Human 6.2 interrogation.

cell cycle changes, propidium iodide was added to RMS cells after $72 \mathrm{~h}$ of 5-aza-dC exposure. Cell cycle distribution analysis in RH30 cells showed a significant accumulation of cells in the G2 phase in 5-aza-dC treated cells compared with controls $(51 . \% \pm 2.1$ vs. $19.9 \% \pm 3.1, \mathrm{p}<$ $0.001)$, while the percentage of cells in the G1 phase decreased by $50 \%(28.4 \% \pm 5.1$ vs. $60.5 \% \pm 3.2, \mathrm{p}<0.01)$ (Figure 6D). Analogous trend was observed in RD cells treated with 5-aza-dC compared with mocked control (G2 phase: $50.2 \% \pm 1.1$ vs. $35.9 \% \pm 1.7, \mathrm{p}<0.01$; G1 phase: $30.1 \% \pm 0.9$ vs. $47.5 \% \pm 1.3, \mathrm{p}<0.01$ ).

These results indicate that demethylation inhibits viability and proliferation of RMS cells due to G2 cell cycle arrest and programmed cell death, and that it might also affect RMS progression by reducing cell migration.

\section{5-aza-dC treatment induces myogenic differentiation}

Finally, to assess whether 5-aza-dC treatment can also promote myogenic differentiation in RMS, RH30 cells were exposed to the demethylating drug followed by assessment of myogenic markers by immunofluorescence experiments. RH30 cells treated with 5 -aza-dC exhibited marked changes in the cytoskeleton structure, as evident by phalloidin staining, and a differentiated phenotype, supported by the MyHC positivity (Figure 6E).

Taken together, these data are partially consistent with those following miR-378a-3p transfection, although the effects in RMS cells are more pronounced, this being due to the fact that 5-aza-dC action is not limited to the regulation of an individual miRNA but that it facilitates the re-expression of several epigenetically silenced genes. 


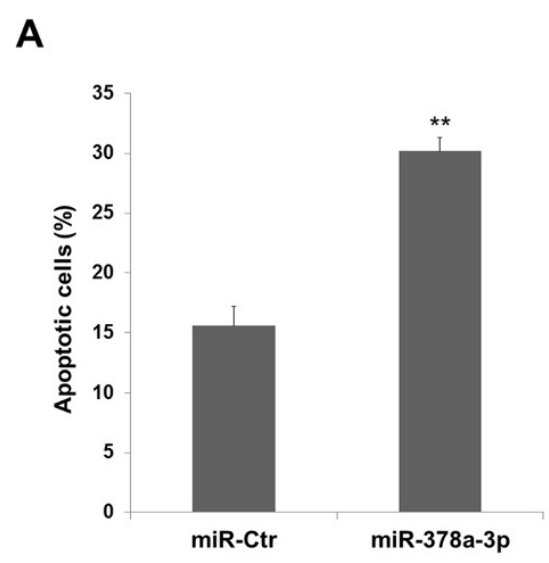

C

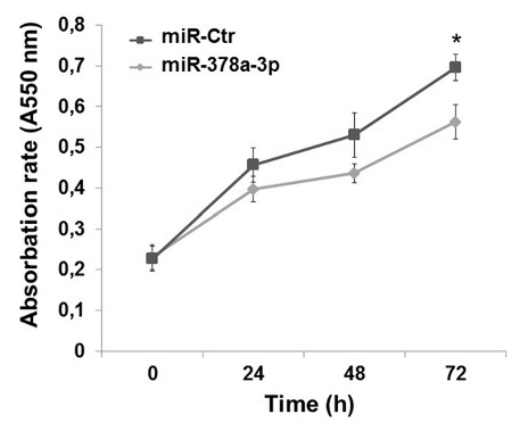

B

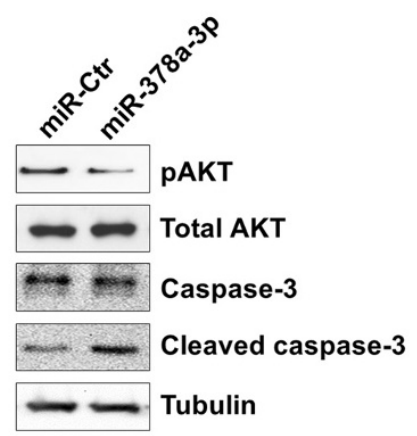

D

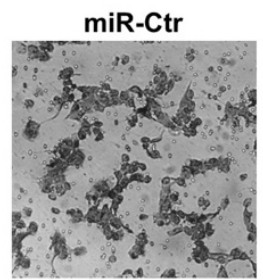
miR-378a-3p
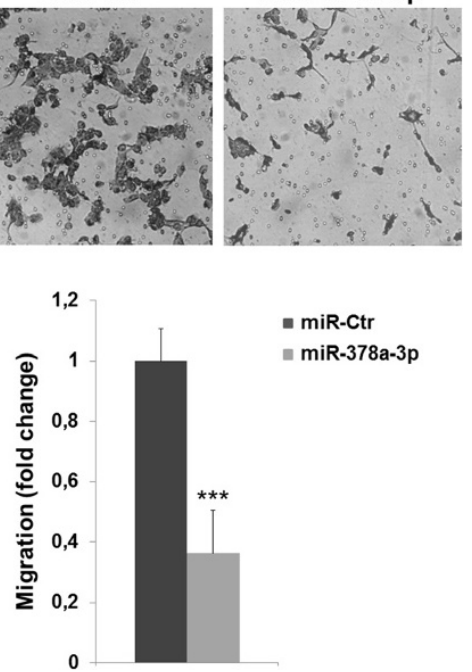

Figure 3 miR-378a-3p involvement in apoptosis, cell proliferation and migration of RMS cells. (A) FACS analysis. Increase of apoptotic cells (Annexin V-positive) in miR-378a-3p compared to miR-Control positive RH30, $72 \mathrm{~h}$ after transfection. Results in the bar chart are the means \pm standard deviation (SD) of three separate experiments. (B) Immunoblot analysis, $72 \mathrm{~h}$ after transfection. Reduction of phosho-AKT (pAKT) levels and up-regulation of cleaved caspase-3 in RH30 positive cells in comparison to miR-Ctr transfected cells. Total AKT andcaspase-3 levels were similar in both samples. Tubulin was used as loading control. (C) MTT assay. Alteration of the cell viability/proliferation rate in miR-378a-3p transfected RH30 compared with mock control cells. Results are means \pm SD of sextuplicate points of two separate experiments. (D) Migration assay. miR-378a-3p transfected RH30 cells compared to negative control cells showed decreased ability to migrate through Boyd chamber membranes. Representative fields of migrated cells through the membrane, stained with crystal violet dye (20x magnification). Data was obtained from the average migration cell number per field $(n=8) \pm S D$ of triplicate experiments performed twice. Asterisks denote data in which miR-378a-3p was significantly different from control $\left(*, p<0.05 ;{ }^{* *}, p<0.01 ; * *, p<0.001\right)$.

\section{Discussion}

RMS is a malignant tumour occurring most commonly in the paediatric population, with a survival of around $20 \%$ when metastatic disease is present at diagnosis [42]. MiRNAs seem to play a crucial role in RMS development and progression, and might represent novel tools for improving the outcome of this malignancy $[12,43,44]$. In this study, the expression levels of miRNAs were measured in a panel of alveolar and embryonal tumours by using deep sequencing technology in order to identify key drivers of RMS specific processes. The global miRNA abundance was reduced in RMS, this contributing to the neoplastic transformation by allowing an increased expression of proteins with oncogenic potential, as previously observed [17]. Notably, many relevant myo-miRNAs, such as miR1, miR-133a and miR-29, were markedly down-regulated in tumour samples in comparison to NSM, as already shown in previous studies carried out by microarray or Q-PCR technologies [21,37]. Interestingly, mir-206 was present with a detectable negative fold change in all comparisons but without being associated with a significant statistical index in the final analysis, as also reported in recent published microarray experiments carried out in the same biological model [45]. MiRNAs expression was 
A

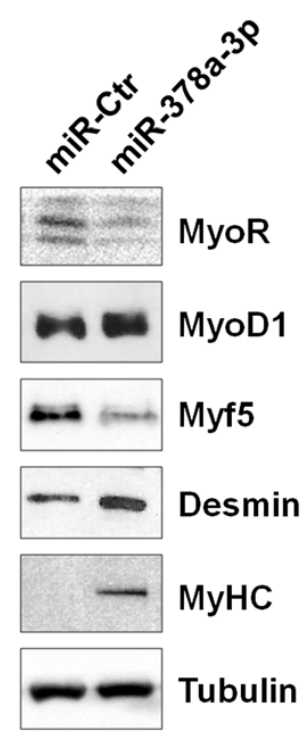

B

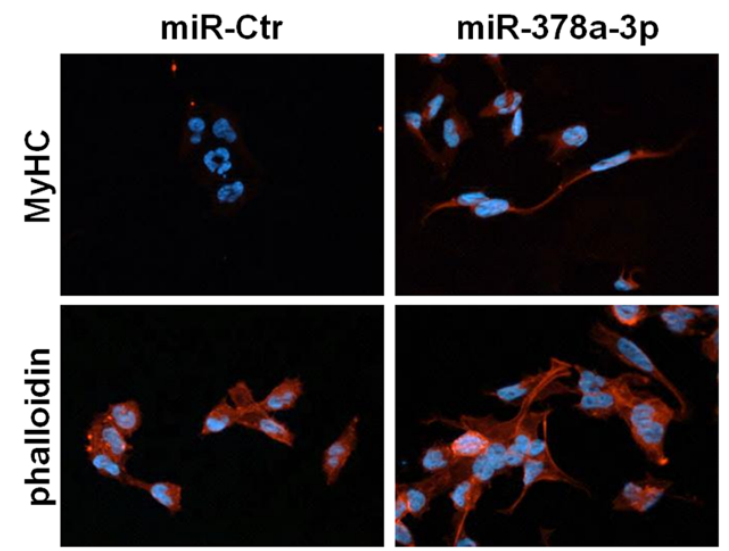

Figure 4 miR-378a-3p activates myogenic differentiation. (A) Immunoblot experiments. RH30 cells transfected with miR-378a-3p mimics or miR-Ctr were analysed for the expression of myogenic differentiation markers. Increased levels of MyoD1 (1.6-foldchange by densitometry), desmin and MyHC proteins and down-regulation of MyoR and Myf5 factors were evident. Tubulin served as protein loading control. (B) Immunofluorescence analysis. MiR-378a-3p ectopic expression evoked noticeable changes in RH30 cell morphology, a more organized actin and myosin arrangement, as visualised by TRITC-phalloidin staining, and a significant positivity for anti-MyHC antibody (100× magnification).

relatively uniform in ARMS and ERMS tumours, and only a restricted number of small regulatory molecules seemed to discriminate the two RMS subtypes. Further analyses need to be undertaken to understand the possible differential role of specific miRNAs and related pathways in ARMS and ERMS oncogenic transformation, and to determine the potential role of miRNAs as diagnostic biomarkers in the two RMS subtypes.

To our knowledge, this is the first study that shows a marked down-regulation of miR-378 family members in RMS. This finding is probably due to the improved specificity and sensitivity of deep sequencing compared to microarray and Q-PCR analyses, deep sequencing also allowing identification of novel MiRNA isoforms. MiR378 molecules were strongly under-expressed both in ARMS and ERMS tumours, this showing that the regulation of these miRNAs does not differentiate among RMS subtypes. Interesting preliminary results showed a significant difference in the abundance of one specific miR378a-3p 3' isoform between RMS and NSM. miR-378 molecules are encoded by different genes but they share identical seed sequences for mRNA target recognition. The most important regulatory small RNA of this family is miR-378a-3p, whose expression is highly variable in different cancers, reflecting the complexity and specificity of the miRNA response [46-48]. In addition, some studies suggest that miR-378a-5p, the opposite strand of miR-378a-3p, also has a functional relevance [49,50]. Our data confirmed that miR-378a-3p was significantly reduced not only in RMS tumours but also in human
RMS cell lines, this finding suggesting a role of miR$378 \mathrm{a}-3 \mathrm{p}$ as a tumour suppressor. Among the main pathways identified as potential targets for miR-378a-3p in our bioinformatics analysis, we chose to focus on IGF1R/AKT signalling, which has a well-established role in cellular growth and survival as well as in myogenic differentiation [40,51,52]. Ectopic expression of miR378a-3p in RH30, an ARMS-derived cell line, markedly reduced IGF1R protein levels and promoted apoptosis via phospho-AKT inhibition and concomitant caspase-3 activation. Furthermore, miR-378a-3p-transfected cells moderately reduced cell proliferation and markedly cell migration. Accordingly, miR-378a-3p has been shown to be involved in carcinogenesis and an anti-oncogenic function has been suggested both in nasopharyngeal [53] and gastric cancer [47] through the negative regulation of VEGF expression, a protein promoting cell migration and inhibiting apoptosis. In a study by Martins et al. [54], RMS development seemed to involve increased IGF1R expression, which in turn enhanced AKT and Bcl-XL-mediated cell survival. The moderate effects on apoptosis and cell growth we observed in RH30 cells could be explained by the presence of the $t(2 ; 13)$ (q35; q14) translocation, since PAX3/FOXO1 chimeric protein is directly involved in the regulation of the IGF1R expression.

In accordance with the observation that in RMS proliferation and migration are inversely related to myogenic differentiation $[3,55]$, we found that miR-378a-3p mimic transfection was also able to promote myogenesis, as 


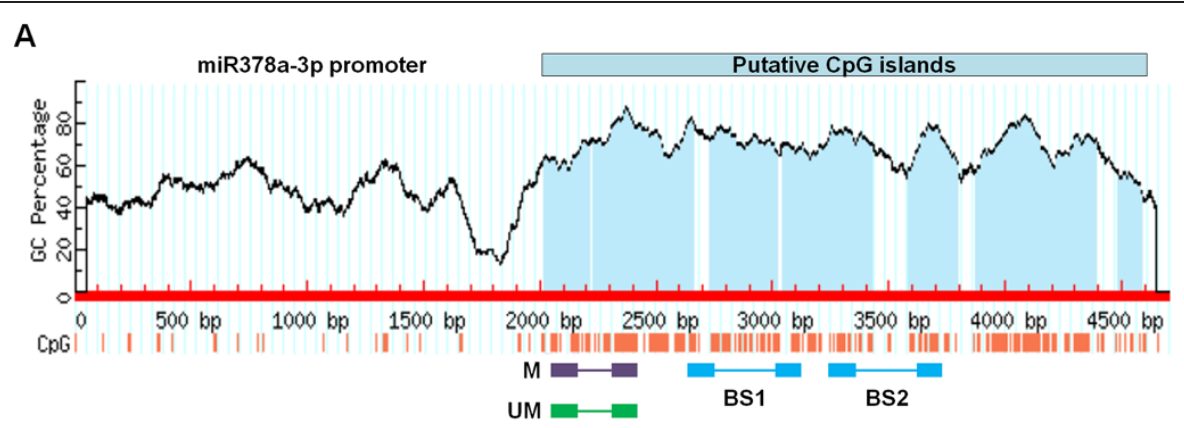

B

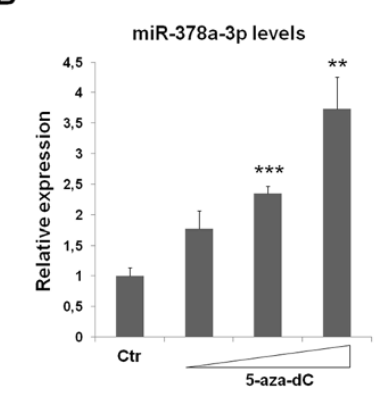

C

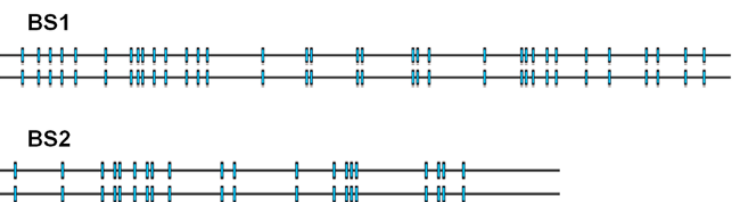

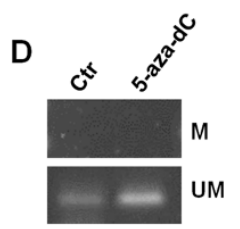

Figure 5 miR-378a-3p response to 5 -aza-dC treatment. (A) Schematic representation of the miR-378a-3p promoter region generated by EMBOSS software. Bioinformatic analysis showed the presence of a high percentage of GC nucleotides and an enriched zone of CpG islands. (B) Effect of increasing doses of 5-aza-dC (2-10-20 $\mu \mathrm{M})$ on mature miR-378-3p levels compared with control RH30 cells $24 \mathrm{~h}$ after treatment. Results are means \pm SD of three separate experiments (**, $p<0.01 ;{ }^{* *} p<0.001$ ). (C) BISMA diagrams showing the methylation level of CpG dinucleotides in the miR-378a promoter in 5-aza-dC and mocked control cells, as revealed by bisulfite sequencing of BS1 and BS2 regions. Blue rectangles indicate non-methylated CpGs. (D) Methylation analysis of miR-378a promoter region by PCR using primer set for methylated (M) or unmethylated (UM) sequences. The presence of a visible UM product indicates that CpG dinucleotides are not methylated in both Ctr and 5-aza-dC-treated cells.

shown by the modulated levels of specific differentiation markers and by the changes in the cell morphology, from star-round to a more elongated appearance. In particular, normalization of miR-378a-3p expression reduced the levels of MyoR, a MyoD1 inhibitor, thus contributing to reactivate myogenesis; this was reflected by the MyHC levels, which is specifically related to committed muscle cells. Furthermore, miR-378a-3p transfected RH30 cells showed a more concentrated assembly of actin bundles, a characteristic of the intermediate muscle differentiation stage [56]. In accordance with these results, recent works have reported miR-378a-3p as a new candidate in skeletal muscle development [37,57]. In C2C12 murine proliferating myoblasts, upmodulation of miR-378 has been proposed as allowing the efficient myotube formation by repressing antagonists of differentiation, like MyoR [57]. Likewise, the expression pattern of miR-378 in porcine longissimus muscles is closely related to myogenesis regulation, mainly with fibre formation [37]. Re-activation of skeletal muscle development in RMS seems to be linked to the IGF1R inhibition, as demonstrated by miR-1 and miR-133a [51,58]. Indeed, the marked down-regulation of miR-378a-3p, miR-1 and miR-133a, and the concomitant up-regulation of IGF1R observed in RMS tumours could explain why cancer cells are prohibited from undergoing terminal differentiation despite their commitment to a myogenic pathway.

This study also underlines the relationship between aberrant epigenetic regulation of tumour suppressors, including miRNAs, and cancer development. The presence of $\mathrm{CpG}$ sites in the promoter regions of the miR378 family members has recently been suggested and partially experimentally demonstrated in other neoplasms [47,59-61]. In our in vitro RMS model, treatment with 5-aza-2'-deoxycytidine, a demethylating agent, was able to restore the expression of mature miR-378a-3p in a dose-dependent manner but without direct effect on DNA methylation status. Therefore, the hypermethylation of $\mathrm{CpG}$ islands at the miR-378a-3p promoter region does not represent a causative mechanism for the marked down-regulation of this miRNA in RMS. 


\section{A}

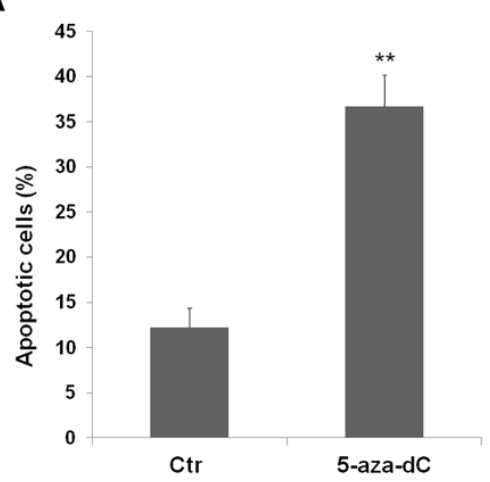

C

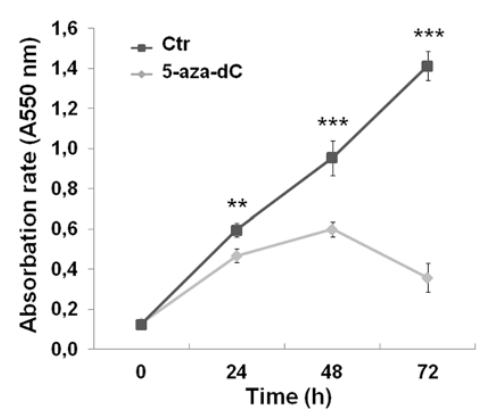

D
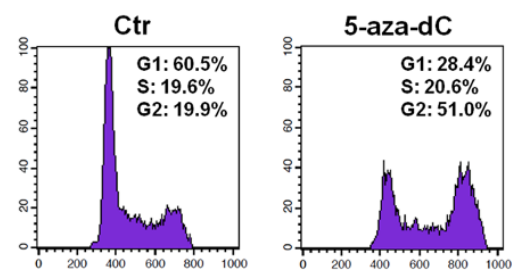

B
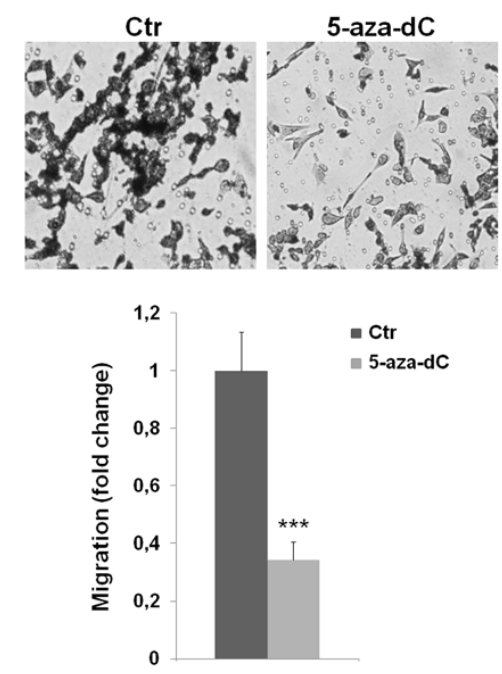

E
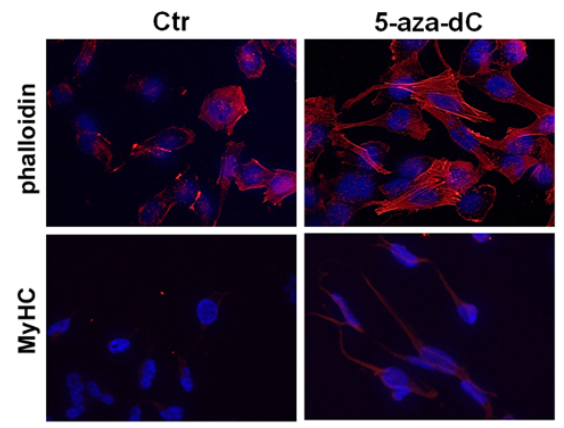

Figure 6 5-aza-dC involvement in apoptosis, cell proliferation, migration and differentiation of RMS cells. (A) FACS analysis. Increased number of apoptotic RH30 cells after treatment with $20 \mu \mathrm{M}$ 5-aza-dC along with paired negative controls (DMSO treatment). Results are the means \pm SD of three separate experiments. (B) Migration assay. 5-aza-dC treated RH30 cells, compared with control, showed significantly impaired ability to cross through Boyd chamber membranes. Representative fields of migrated cells through the membrane, stained with crystal violet dye (20x magnification). (C) MTT assay. Time-dependent inhibition of cell proliferation after 5-aza-dC treatment resulted in $80 \%$ decrease at 72 h. Data are shown as means \pm SD of sextuplicate points of two separate experiments. (D) FACS analysis. Cell cycle distribution of $20 \mu \mathrm{M} 5$-aza-dC RH30 treated cells showed a marked arrest in G2-phase after $72 \mathrm{~h}$. Results are means \pm SD of three separate experiments. (E) Immunofluorescence analysis. Five-aza-dC exposure led to marked changes of RH30 cell morphology, to more organised actin bundles (TRITC-phalloidin staining) and to MyHC positivity (100x magnification). Asterisks denote data in which miR-378a-3p was significantly different from control $(* *, p<0.01$; ***, $p<0.001)$.

Indeed, many $\mathrm{CpG}$ islands located at genes that have a tissue-restricted expression pattern, can remain methylation-free even when their associated genes are silent. Inactive unmethylated $\mathrm{CpG}$ island promoters show elevated levels of dimethylation at histone H3 lysine 4 (H3K4), suggesting that this chromatin mark may protect DNA from methylation [62]. In line with this model, recruitment of H3K4 methylases by unmethylated CpGs has recently been suggested [63]. Notwithstanding, our data imply that epigenetic modifications may play an indirect role on
miR-378a expression by acting on specific transcription factors able to bind the miR-378a promoter region, which also warrant further study. Consistent with the up-regulation of miR-378a-3p levels, 5-aza-dC exposure affected apoptosis, proliferation and migration of RMS cells, with more dramatic effects. In particular, the resulting DNA hypomethylation arrested the cell cycle at G2-phase with a concomitant induction of myogenic differentiation, as demonstrated by the more elongated cell morphology and enhanced MyHC expression. Most likely, the fact that azacytidine has a much broader effect on the 
RH30 cellular phenotype reflects the fact that the transcriptional activation is not limited to miR-378a-3p but involves several epigenetically silenced genes. Aberrant DNA methylation and histone deacetylation was reported to have a key role in silencing plakoglobin gene expression in ARMS cells, whose over-expression was shown to suppress tumorigenicity in different cancer cell lines [64]. Moreover, recent findings suggest that suppression of BMP2 by epigenetic silencing may play a critical role in the genesis of RMS [65]. Based on these findings, 5-aza$\mathrm{dC}$ might be a promising therapeutic agent in patients with RMS. To date, azacytidine is FDA-approved for the treatment of patients with acute myeloid leukemia and myelodysplastic syndrome [66,67]. Data in sarcomas are still limited [68], but ongoing clinical trials in the field of epigenetic treatment suggest that this approach may be a potential option for patients with sarcomas, including RMS [69].

\section{Conclusions}

This study suggests that miR-378a-3p is involved in RMS pathogenesis, exerting tumour suppressor-like activity. Since miR-378a-3p is down-regulated in other malignancies, the identification of the regulated tumorigenic pathways might have important implications in oncology. A better understanding of the biological mechanisms underlying RMS development represents a fundamental step in order to identify novel diagnostic and prognostic markers, as well as to develop targeted therapies in children and adolescents with RMS. Indeed, a combination of molecules inhibiting IGF1R signalling and promoting demethylation might be a possible therapeutic strategy worth to be further investigated in this tumour.

\section{Additional files}

Additional file 1: Table S1. Expression of miR-378 family members in RMS tumours. (A) miR-378 molecules differentially expressed in ARMs vs. NMS, with NMS baseline. (B): miR-378 molecules differentially expressed in ERMS vs. NMS, with NMS baseline. The members of the miR-378 family have $F C$ values always negative and significant in both comparisons, indicating a strong under-expression in the investigated ARMS and ERMS samples.

Additional file 2: Figure S1. Validation of miRNA array data by quantitative real-time polymerase chain reaction (Q-PCR). (A) Relative expression levels for miR-378a-3p, miR-378a-5p, miR-483-3p and miR-503-5p in RMS patients (ARMS and ERMS collectively considered) in comparison to NSM. Histograms indicate the mean value \pm SD of independent samples (ARMS1-2-3-4-7-36-37 and ERMS1-2-3-4-12-21-23-27); (B) Relative fold change of miR-378a-3p, miR-378a-5p, miR-483-3p and miR-503-5p in RMS cell lines in comparison to NSM. Histograms represent the mean value \pm SD of 4 different cell lines (RH4, RH30, RD and RD18). Y-axis values are expressed as $\log _{2} \mathrm{FC}\left({ }^{* *}, \mathrm{p}<0.05 ;{ }^{* *} \mathrm{p}<0.01\right)$.

Additional file 3: Table S2. Description of worksheet. Target Genes Gene Annotation => ENSEMBL annotation for miR-378a-3p target genes. Target Genes GO Annotation => ENSEMBL GO annotations for miR-378a-3p target genes; cancer- and muscle- associated categories are highlighted in blue. TGT Genes Reactome Enrichment $=>$ analysis of over representation of Reactome Pathways in the miR-378a-3p target genes. TGT Genes DAVID Clustering = > most significant functional clusters from a DAVID analysis of the miR-378a-3p target genes. TGT Genes FI Network no linkers $=>$ graphical representation and description of the Functional Interactions associated with the network edges of a Fl network analysis performed on the miR-378a-3p target genes. No linker genes (functional links not present in the original gene list) were considered in this analysis. TGT Genes Fl Network - Pathways = > pathway (CellMap, Reactome, KEGG, NCl Panther and BioCarta) enrichment analysis of the FI network. Fl Network GO Enrichment $=>$ Graphical representation and table of the most significant results of a Network Ontology Analysis on the FI network, keeping into account the functional links (edges) between the network nodes. Fl Network GO (Cellular Component, Biological Process, Molecular Function) $=>$ enrichment analysis, category by category, of the GO annotation associated with the FI network. TGT Genes Clustered FI Network = > cluster (module) analysis of the Fl network, searching for functional modules and associated pathway over representation. Clustered Fl Network + Linkers = > graphical representation and analysis of the over represented pathways associated with the network edges of a (clustered) FI network analysis performed on the miR-378a-3p target genes. Linker genes (functional links not present in the original gene list) were considered in this analysis, so as to give a broader view of the functional significance of the identified target gene set.

Additional file 4: Figure S2. Levels of transfected miR-378a-3p mature mimics in RMS cell lines. Relative expression of miR-378a-3p by Q-PCR at $72 \mathrm{~h}$ post transfection in RH30 and RD cells compared with miR-Ctr transfected cells. Levels of miR-378a-5p were measured to confirm the specificity of miR-378a-3p mimic transfection. Three independent experiments were performed. Comparing with respective miR-Ctr, ${ }^{* *} p<0.01$.

\section{Competing interests}

The authors declare that they have no competing interest.

\section{Authors' contributions}

FM designed and performed experiments, analysed data and wrote the manuscript. SC designed and performed experiments, analysed data and made figures. HPMD provided tumour samples and clinical information, and critically revised the manuscript. AF analysed deep sequencing data and realised summary tables and graphics. SCa performed experiments. AG performed statistical analyses of deep sequencing data, interrogated bioinformatics tools and contributed to write methods and results. BP, AC and ADG provided clinical information. AP contributed new reagents and edited the manuscript. AM contributed new reagents and edited the manuscript. CD provided tumour samples and clinical information, contributed new reagents, analysed data and critically revised the manuscript. All authors read and approved the manuscript before submission.

\section{Acknowledgements}

This work was partially supported by "Associazione Onlus Fabrizio Procaccini", "Io... domani, Associazione Onlus per la Lotta contro i Tumori Infantili" (ALTI), "Isle of Man Anti-Cancer Association", Italian "Ministero dell'Istruzione, dell'Università e della Ricerca" (MIUR), and Genomnia srl R\&D funds. The Authors are grateful to: Dr. David N. Shapiro for providing RH4 and RH3O ARMS cell lines; Prof. Pier Luigi Lollini for providing RD and RD18 ERMS cell lines; Ms. Olga Mannarino for technical assistance; Dr. Simona Ceccarelli and Dr. Saula Checquolo for Apotom image acquisition; and Dr. Bettina Nadorp for useful discussions on miR-378 family promoter characterisation.

\section{Author details}

'Department of Paediatrics and Infantile Neuropsychiatry, Sapienza University of Rome, Viale Regina Elena 324, 00161 Rome, Italy. ${ }^{2}$ Present address: Department of Molecular Medicine, Sapienza University of Rome, Viale Regina Elena 324, 00161 Rome, Italy. ${ }^{3}$ Department of Oncology, Alder Hey Children's NHS Foundation Trust, Eaton Road, L12 2AP Liverpool, United Kingdom. ${ }^{4}$ School of Reproductive and Developmental Medicine, University of Liverpool, L12 2AP Liverpool, United Kingdom. ${ }^{5}$ Genomnia srl, Via Nerviano 31/b, 20020 Lainate, MI, Italy. ${ }^{6}$ Department of Experimental Medicine, Sapienza University of Rome, Viale Regina Elena 324, 00161 Rome, Italy. 
Received: 19 May 2014 Accepted: 4 November 2014

Published: 25 November 2014

\section{References}

1. McDowell HP: Update on childhood rhabdomyosarcoma. Arch Dis Child 2003, 88(4):354-357.

2. Harms D: New entities, concepts, and questions in childhood tumor pathology. Gen Diagn Pathol 1995, 141(1):1-14.

3. Merlino G, Helman LJ: Rhabdomyosarcoma: working out the pathways. Oncogene 1999, 18(38):5340-5348.

4. Smith AC, Squire JA, Thorner P, Zielenska M, Shuman C, Grant R, Chitayat D, Nishikawa JL, Weksberg R: Association of alveolar rhabdomyosarcoma with the Beckwith-Wiedemann syndrome. Pediatr Dev Pathol 2001, 4(6):550-558.

5. Yang P, Grufferman S, Khoury MJ, Schwartz AG, Kowalski J, Ruymann FB, Maurer HM: Association of childhood rhabdomyosarcoma with neurofibromatosis type I and birth defects. Genet Epidemio/ 1995, 12(5):467-474

6. Trahair T, Andrews L, Cohn RJ: Recognition of Li Fraumeni syndrome at diagnosis of a locally advanced extremity rhabdomyosarcoma. Pediatr Blood Cancer 2007, 48(3):345-348

7. Barr FG: Gene fusions involving PAX and FOX family members in alveolar rhabdomyosarcoma. Oncogene 2001, 20(40):5736-5746.

8. Lagutina I, Conway SJ, Sublett J, Grosveld GC: Pax3-FKHR knock-in mice show developmental aberrations but do not develop tumors. Mol Cell Biol 2002, 22:7204-7216.

9. Galindo RL, Allport JA, Olson EN: A Drosophila model of the rhabdomyosarcoma initiator PAX7-FKHR. Proc Natl Acad Sci U S A 2006 103:13439-13444

10. Xia SJ, Pressey JG, Barr FG: Molecular pathogenesis of rhabdomyosarcoma. Cancer Biol Ther 2002, 1(2):97-104

11. Armeanu-Ebinger S, Herrmann D, Bonin M, Leuschner I, Warmann SW, Fuchs J, Seitz G: Differential expression of miRNAs in rhabdomyosarcoma and malignant rhabdoid tumor. Exp Cell Res 2012, 318(20):2567-2577.

12. Novak J, Vinklarek J, Vasku JB, Slaby O: MicroRNAs involved in skeleta muscle development and their roles in rhabdomyosarcoma pathogenesis. Pediatr Blood Cancer 2013, 60(11):1739-1746.

13. Valencia-Sanchez MA, Liu J, Hannon GJ, Parker R: Control of translation and mRNA degradation by miRNAs and siRNAs. Genes Dev 2006, 20(5):515-524.

14. Winter J, Jung S, Keller S, Gregory Rl, Diederichs S: Many roads to maturity: microRNA biogenesis pathways and their regulation. Nat Cell Biol 2009, 11(3):228-234

15. Bartel DP: MicroRNAs: genomics, biogenesis, mechanism, and function. Cell 2004, 116(2):281-297.

16. Lewis BP, Burge CB, Bartel DP: Conserved seed pairing, often flanked by adenosines, indicates that thousands of human genes are microRNA targets. Cell 2005, 120(1):15-20.

17. Lu J, Getz G, Miska EA, Alvarez-Saavedra E, Lamb J, Peck D, Sweet-Cordero A, Ebert BL, Mak RH, Ferrando AA, Downing JR, Jacks T, Horvitz HR, Golub TR: MicroRNA expression profiles classify human cancers. Nature 2005, 435(7043):834-838.

18. Calin GA, Croce CM: MicroRNA signatures in human cancers. Nat Rev Cancer 2006, 6(11):857-866.

19. Wei JS, Johansson P, Chen QR, Song YK, Durinck S, Wen X, Cheuk AT, Smith MA, Houghton P, Morton C, Khan J: microRNA profiling identifies cancer-specific and prognostic signatures in pediatric malignancies. Clin Cancer Res 2009, 15(17):5560-5568.

20. Subramanian S, Lui WO, Lee CH, Espinosa I, Nielsen TO, Heinrich MC, Corless CL, Fire AZ, van de Rijn M: MicroRNA expression signature of human sarcomas. Oncogene 2008, 27(14):2015-2026

21. Missiaglia E, Shepherd CJ, Patel S, Thway K, Pierron G, Pritchard-Jones K, Renard M, Sciot R, Rao P, Oberlin O, Delattre O, Shipley J: MicroRNA-206 expression levels correlate with clinical behavior of rhabdomyosarcomas. Br J Cancer 2010, 102(12):1769-1777.

22. Taulli R, Bersani F, Foglizzo V, Linari A, Vigna E, Ladanyi M, Tuschl T, Ponzetto C: The muscle-specific microRNA miR-206 blocks human rhabdomyosarcoma growth in xenotransplanted mice by promoting myogenic differentiation. J Clin Invest 2009, 119(8):2366-2378.

23. Sun MM, Li JF, Guo LL, Xiao HT, Dong L, Wang F, Huang FB, Cao D, Qin T, Yin XH, Li JM, Wang SL: TGF- $\beta 1$ suppression of microRNA-450b-5p expression: a novel mechanism for blocking myogenic differentiation of rhabdomyosarcoma. Oncogene. in press.

24. Huang HJ, Liu J, Hua H, Li SE, Zhao J, Yue S, Yu TT, Jin YC, Cheng SY: MiR-214 and N-ras regulatory loop suppresses rhabdomyosarcoma cell growth and xenograft tumorigenesis. Oncotarget 2014, 5(8):2161-2175.

25. Egger $G$, Liang $G$, Aparicio A, Jones PA: Epigenetics in human disease and prospects for epigenetic therapy. Nature 2004, 429(6990):457-463.

26. Jones PA: DNA methylation and cancer. Oncogene 2002, 21(35):5358-5360.

27. Lujambio A, Ropero S, Ballestar E, Fraga MF, Cerrato C, Setien F, Casado S, Suarez-Gauthier A, Sanchez-Cespedes M, Git A, Spiteri I, Das PP, Caldas C, Miska E, Esteller M: Genetic unmasking of an epigenetically silenced microRNA in human cancer cells. Cancer Res 2007, 67(7):1424-1429.

28. Diao Y, Guo X, Jiang L, Wang G, Zhang C, Wan J, Jin Y, Wu Z: miR-203, a tumor suppressor frequently down-regulated by promoter hypermethylation in rhabdomyosarcoma. J Biol Chem 2014, 289:529-539.

29. Maurer HM, Crist W, Lawrence W, Ragab AH, Beverly Raney R, Webber B, Wharam M, Vietti TJ, Beltangady M, Gehan EA, Hammond D, Hays DM, Heyn R, Newton W, Ortega J, Ruymann FB, Soule E, Tefft M: The Intergroup Rhabdomyosarcoma Study-I. A final report. Cancer 1988, 61(2):209-220.

30. Griffiths-Jones S, Saini HK, van Dongen S, Enright AJ: miRBase: tools for microRNA genomics. Nucleic Acids Res 2008, 36(Database issue):D154-D158.

31. Robinson MD, Oshlack A: A scaling normalization method for differential expression analysis of RNA-seqdata. Genome Biol 2010, 11(3):R25.

32. Robinson MD, Smyth GK: Small-sample estimation of negative binomial dispersion, with applications to SAGE data. Biostatistics 2008, 9(2):321-332.

33. Dweep H, Sticht C, Pandey P, Gretz N: miRWalk-database: prediction of possible miRNA binding sites by "walking" the genes of three genomes. J Biomed Inform 2011, 44(5):839-847.

34. Wu G, Feng $X$, Stein L: A human functional protein interaction network and its application to cancer data analysis. Genome Biol 2010, 11(5):R53.

35. Wang J, Huang Q, Liu ZP, Wang Y, Wu LY, Chen L, Zhang XS: NOA: a novel network ontology analysis method. Nucleic Acids Res 2011, 39(13):e87.

36. Rao PK, Missiaglia E, Shields L, Hyde G, Yuan B, Shepherd CJ, Shipley J, Lodish HF: Distinct roles for miR-1 and miR-133a in the proliferation and differentiation of rhabdomyosarcoma cells. FASEB J 2010, 24(9):3427-3437.

37. Hou X, Tang Z, Liu H, Wang N, Ju H, Li K: Discovery of MicroRNAs associated with myogenesis by deep sequencing of serial developmental skeletal muscles in pigs. PLoS One 2012, 7(12):e52123.

38. LeRoith D, Werner H, Neuenschwander S, Kalebic T, Helman LJ: The role of the insulin-like growth factor-I receptor in cancer. Ann N Y Acad Sci 1995, 766:402-408.

39. Knezevic I, Patel A, Sundaresan NR, Gupta MP, Solaro RJ, Nagalingam RS, Gupta M: A novel cardiomyocyte-enriched microRNA, miR-378, targets insulin-like growth factor 1 receptor: implications in postnatal cardiac remodeling and cell survival. J Biol Chem 2012, 287(16):12913-12926.

40. Ganesan J, Ramanujam D, Sassi Y, Ahles A, Jentzsch C, Werfel S, Leierseder S, Loyer X, Giacca M, Zentilin L, Thum T, Laggerbauer B, Engelhardt S: MiR-378 controls cardiac hypertrophy by combined repression of mitogen-activated protein kinase pathway factors. Circulation 2013, 127(21):2097-2106.

41. Li H, Dai S, Zhen T, Shi H, Zhang F, Yang Y, Kang L, Liang Y, Han A: Clinical and biological significance of miR-378a-3p and miR-378a-5p in colorectal cancer. Eur J Cancer 2014, 50(6):1207-1221.

42. McDowell HP, Foot AB, Ellershaw C, Machin D, Giraud C, Bergeron C: Outcomes in paediatric metastatic rhabdomyosarcoma: results of The International Society of Paediatric Oncology (SIOP) study MMT-98. Eur $J$ Cancer 2010, 46(9):1588-1595.

43. Fabbri M: MicroRNAs and cancer: towards a personalized medicine. Curr Mol Med 2013, 13(5):751-756

44. Tan S, Wu Y, Zhang CY, Li J: Potential MicroRNA targets for cancer chemotherapy. Curr Med Chem 2013, 20(29):3574-3581.

45. Li L, Sarver AL, Alamgir S, Subramanian S: Downregulation of microRNAs miR-1, -206 and -29 stabilizes PAX3 and CCND2 expression in rhabdomyosarcoma. Lab Invest 2012, 92(4):571-583.

46. Chen LT, Xu SD, Xu H, Zhang JF, Ning JF, Wang SF: MicroRNA-378 is associated with non-small cell lung cancer brain metastasis by promoting cell migration, invasion and tumorangiogenesis. Med Oncol 2012, 29(3):1673-1680

47. Deng H, Guo Y, Song H, Xiao B, Sun W, Liu Z, Yu X, Xia T, Cui L, Guo J: MicroRNA-195 and microRNA-378 mediate tumor growth suppression by epigenetical regulation in gastric cancer. Gene 2013, 518(2):351-359. 
48. Novello C, Pazzaglia L, Cingolani C, Conti A, Quattrini I, Manara MC, Tognon $M$, Picci P, Benassi MS: miRNA expression profile in human osteosarcoma: role of miR-1 and miR-133b in proliferation and cell cycle control. Int J Oncol 2013, 42(2):667-675.

49. Eichner LJ, Perry MC, Dufour CR, Bertos N, Park M, St-Pierre J, Giguere V: miR-378 $\left.{ }^{*}\right)$ mediates metabolic shift in breast cancer cells via the PGC-1/ ERR transcriptional pathway. Cell Metab 2010, 12(4):352-361.

50. Carrer M, Liu N, Grueter CE, Williams AH, Frisard Ml, Hulver MW, Bassel-Duby $\mathrm{R}$, Olson EN: Control of mitochondrial metabolism and systemic energy homeostasis by microRNAs 378 and 378*. Proc Natl Acad Sci U S A 2012, 109(38):15330-15335.

51. Huang MB, Xu H, Xie SJ, Zhou H, Qu LH: Insulin-like growth factor-1 receptor is regulated by microRNA-133 during skeletal myogenesis. PLoS One 2011, 6(12):e29173.

52. Farhana L, Dawson MI, Murshed F, Das JK, Rishi AK, Fontana JA: Upregulation of miR-150* and miR-630 induces apoptosis in pancreatic cancer cells by targeting IGF-1R. PLoS One 2013, 8(5):e61015.

53. Hua Z, Lv Q, Ye W, Wong CK, Cai G, Gu D, Ji Y, Zhao C, Wang J, Yang BB, Zhang $Y$ : MiRNA-directed regulation of VEGF and other angiogenic factors under hypoxia. PLOS One 2006, 1:e116.

54. Martins AS, Olmos D, Missiaglia E, Shipley J: Targeting the insulin-like growth factor pathway in rhabdomyosarcomas: rationale and future perspectives. Sarcoma 2011, 2011:209736.

55. Lollini PL, De Giovanni C, Landuzzi L, Nicoletti G, Scotlandi K, Nanni P: Reduced metastatic ability of in vitro differentiated human rhabdomyosarcoma cells. Invasion Metastasis 1991, 11(2):116-124.

56. Burattini S, Ferri P, Battistelli M, Curci R, Luchetti F, Falcieri E: C2C12 murine myoblasts as a model of skeletal muscle development: morpho-functional characterization. Eur J Histochem 2004, 48(3):223-233.

57. Gagan J, Dey BK, Layer R, Yan Z, Dutta A: MicroRNA-378 targets the myogenic repressor MyoR during myoblast differentiation. J Biol Chem 2011, 286(22):19431-19438.

58. Elia L, Contu R, Quintavalle M, Varrone F, Chimenti C, Russo MA, Cimino V, De Marinis L, Frustaci A, Catalucci D, Condorelli G: Reciprocal regulation of microRNA-1 and insulin-like growth factor-1 signal transduction cascade in cardiac and skeletal muscle in physiological and pathological conditions. Circulation 2009, 120(23):2377-2385.

59. Monteys AM, Spengler RM, Wan J, Tecedor L, Lennox KA, Xing Y, Davidson BL: Structure and activity of putative intronic miRNA promoters. RNA 2010, 16(3):495-505.

60. Li Z, Gu X, Fang Y, Xiang J, Chen Z: microRNA expression profiles in human colorectal cancers with brain metastases. Oncol Lett 2012, 3(2):346-350.

61. Scapoli L, Palmieri A, Lo Muzio L, Pezzetti F, Rubini C, Girardi A, Farinella F, Mazzotta M, Carinci F: MicroRNA expression profiling of oral carcinoma identifies new markers of tumorprogression. Int J Immunopathol Pharmacol 2010, 23(4):1229-1234.

62. Weber M, Hellmann I, Stadler MB, Ramos L, Pääbo S, Rebhan M, Schübeler D: Distribution, silencing potential and evolutionary impact of promote DNA methylation in the human genome. Nat Genet 2007, 39(4):457-466.

63. Ayton PM, Chen EH, Cleary ML: Binding to nonmethylated CpG DNA is essential for target recognition, transactivation, and myeloid transformation by an MLL oncoprotein. Mol Cell Biol 2004, 24:10470-10478.

64. Gastaldi T, Bonvini P, Sartori F, Marrone A, Iolascon A, Rosolen A: Plakoglobin is differentially expressed in alveolar and embryonalrhabdomyosarcoma and is regulated by DNA methylation and histone acetylation. Carcinogenesis 2006, 27(9):1758-1767.

65. Wolf S, Hagl B, Kappler R: Identification of BMP2 as an epigenetically silenced growth inhibitor in rhabdomyosarcoma. Int J Oncol 2014, 44(5):1727-1735.

66. Santini V, Kantarjian HM, Issa JP: Changes in DNA methylation in neoplasia: pathophysiology and therapeutic implications. Ann Intern Med 2001, 134(7):573-586.

67. Fenaux P, Mufti GJ, Hellstrom-Lindberg E, Santini V, Gattermann N, Germing U, Sanz G, List AF, Gore S, Seymour JF, Dombret H, Backstrom J, Zimmerman L, McKenzie D, Beach CL, Silverman LR: Azacitidine prolongs overall survival compared with conventional care regimens in elderly patients with low bone marrow blast count acute myeloid leukemia. J Clin Oncol 2010, 28(4):562-569.

68. Hurtubise A, Bernstein ML, Momparler RL: Preclinical evaluation of the antineoplastic action of 5-aza-2'-deoxycytidine and different histone deacetylase inhibitors on human Ewing's sarcoma cells. Cancer Cell Int 2008, 8:16.

69. Cote GM, Choy E: Role of Epigenetic Modulation for the Treatment of Sarcoma. Curr Treat Options Oncol 2013, 14(3):454-464.

doi:10.1186/1471-2407-14-880

Cite this article as: Megiorni et al.: Deep Sequencing the microRNA profile in rhabdomyosarcoma reveals down-regulation of miR-378 family members. BMC Cancer 2014 14:880.

\section{Submit your next manuscript to BioMed Central and take full advantage of:}

- Convenient online submission

- Thorough peer review

- No space constraints or color figure charges

- Immediate publication on acceptance

- Inclusion in PubMed, CAS, Scopus and Google Scholar

- Research which is freely available for redistribution

Submit your manuscript at www.biomedcentral.com/submit
C) BioMed Central 